\title{
Chapter 3 \\ Composition, Separation of Roles and Model-Driven Approaches as Enabler of a Robotics Software Ecosystem
}

\author{
Christian Schlegel, Alex Lotz, Matthias Lutz, and Dennis Stampfer
}

\begin{abstract}
Successful engineering principles for building software systems rely on the separation of concerns for mastering complexity. However, just working on different concerns of a system in a collaborative way is not good enough for economically feasible tailored solutions. A successful approach for this is the composition of complex systems out of commodity building blocks. These come as is and can be represented as blocks with ports via data sheets. Data sheets are models and allow a proper selection and configuration as well as the prediction of the behavior of a building block in a specific context. This chapter explains how model-driven approaches can be used to support separation of roles and composition for robotics software systems. The models, open-source tools, open-source robotics software components and fully deployable robotics software systems shape a robotics software ecosystem.
\end{abstract}

\section{Aims and Challenges of Software Engineering for Robotics}

Many definitions refer to a robot as a machine that is able to perform a variety of tasks and that is reprogrammable in order to become multifunctional. Thus, as soon as a machine reaches some level of flexibility and versatility in its applicability, we call it a robot. In this way, the most advanced robots are called service robots. A service robot shall not only be able to fulfill a multitude of different tasks. It is expected to do this successfully and with some robustness even under varying circumstances, as it is quite normal in open-ended environments and when a robot needs to share its workspace with others [1].

\footnotetext{
C. Schlegel $(\bowtie) \cdot$ A. Lotz $\cdot$ M. Lutz $\cdot$ D. Stampfer

Technische Hochschule Ulm, Ulm, Germany

e-mail: christian.schlegel@thu.de; alex.lotz@thu.de; matthias.lutz@thu.de;

dennis.stampfer@thu.de

http://www.servicerobotik-ulm.de
}

(C) The Author(s) 2021

A. Cavalcanti et al. (eds.), Software Engineering for Robotics, https://doi.org/10.1007/978-3-030-66494-7_3 
The aspect of reprogramming is deeply rooted in the notion of a robot as is the challenge of making its flexibility exploitable. In the ideal case, that can be done with as little effort as possible and by the user itself. Of course, there is the full spectrum from simple robots (automated devices) to autonomous systems (making decisions on their own and being able to revise decisions), but software is an indispensable tool for implementing and operating all of them.

The question now is whether Software Engineering for Robotics is different from software engineering for other domains or whether it is different with respect to software engineering in general [2]. The short answer is no, there is nothing special at all about Software Engineering for Robotics.

At first glance, that statement seems to be contradictory to the daily experience: it often ends up in a costly IT project or software project when integrating a robot into existing infrastructure or when tailoring a robot to a new use case. Studies identified software as the road block (or at least as the bottleneck) in bringing robots to new application domains [3, 4]. Indeed, Software Engineering for Robotics needs to be mastered in a way that is economically feasible for the application domains so that the flexibility of robots can be exploited.

Figure 3.1 outlines a perspective for a business ecosystem for robotics software systems. Our aim is to introduce into the domain of robotics software the mechanisms and structures of a business ecosystem to let robotics exploit all the benefits of value networks. In this section, we first give an introduction into business
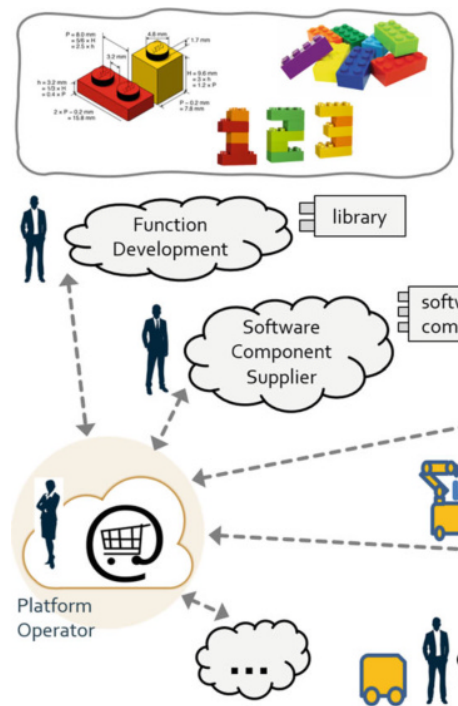

brary
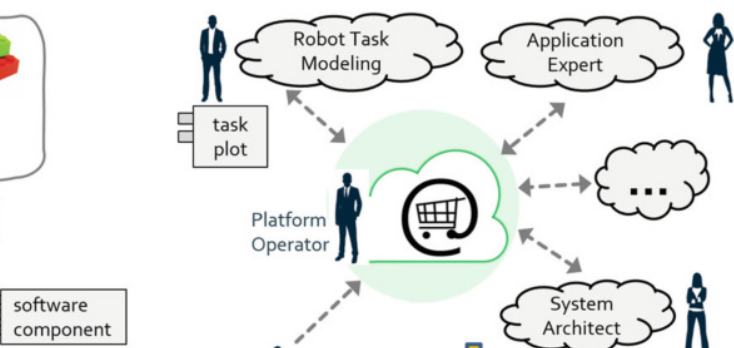

plot
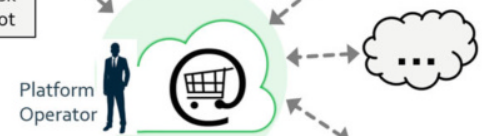

Operator
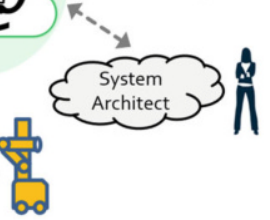

Fig. 3.1 Software engineering is about mastering interfaces between building blocks, competencies and responsibilities. Properly identified and agreed interfaces are the enabler for composition and for separation of roles and thus for a robotics software business ecosystem that comes with marketplaces for composable assets. Building complex software systems for robotics can become as easy as building with Lego bricks. 
ecosystems and into software technologies used in robotics. The subsequent sections then outline how to arrange software technologies used in robotics to shape a robotics software business ecosystem.

\subsection{Carving Out the Specifics of Software for Robotic Systems}

Unfortunately, markets become more and more volatile, products have shorter market life time, and the ongoing trend to customization is another driver that further increases the number of product variants [5]. Quantities of the same product are not high enough anymore to ensure the return of investment of highly specialized and most advanced single-purpose machines via a single product.

Thus, standardized automation solutions able to handle a single scenario are not sufficient anymore. An expensive machine either must cope with a product mix (flexibility), or the effort for adjusting it to the next scenario must be low enough (adaptability). Here is where users expect that robots are such kinds of machines: (1) flexible and adaptable and (2) matching the high demands in fulfilling complex manufacturing steps or whatever other tasks, even in workspaces shared with others or even when robust task fulfillment requires at least a minimum level of autonomy.

Right now, one can basically go for the offered families of standardized robots that come with a reasonable price tag. However, there are many scenarios that one cannot address with them. They are limited in flexibility and adaptability-very often so limited that one faces problems in properly addressing a specific scenario. On the other hand, custom-made robots are also not yet an option as they are still far from being economically feasible under the constraints of an application domain. Although they can be made to perfectly fit requirements, this is then priced accordingly, and such a robot nowadays still does not fulfill the level of flexibility and adaptability which one expects from it to get on the safe side for a return of investment [6].

To summarize, the changeability and flexibility of robotic systems often is not yet economically exploitable to face the pressure of lot-size one. Robots are in many cases not yet a tool that users can adjust themselves to match their daily changing needs. In robotics, costs of changes are not in proper relation to the similarity of an existing solution (Fig. 3.2). Unfortunately, expectations in many application domains where robotics can contribute cannot yet be fulfilled due to high efforts and costs.

The challenge is nothing less than coming up with a different way of building robotic systems. Otherwise, the pull from application domains for robotic solutions helping the application domains to reach their next level of automation cannot be fulfilled. Robots need to make progress in fulfilling the promise of being flexible and adaptable machines. Indeed, software and software tools play a major role in implementing functionalities of robots, in enabling robots to perform more advanced tasks and in empowering users to command robots to their benefits. Thus, one cannot help but admit that the current practice of how we build and manage robotics software systems needs to change in order to address the above-outlined challenges. 


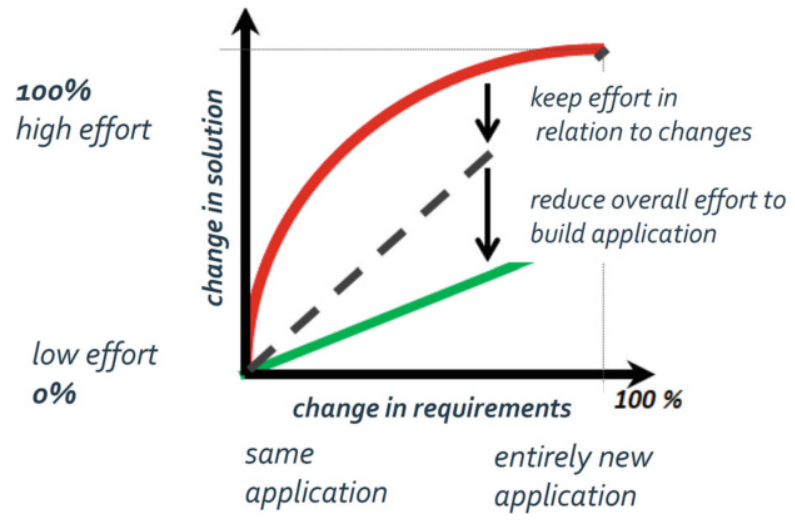

Fig. 3.2 In robotics, costs of changes are not in proper relation to the similarity of an already existing solution. This needs to be resolved in order to enable the widespread use of robotics.

A different way of building robotic systems should tackle questions like: (1) Can we reason about robotic systems before we build them? (2) Can we answer what-if questions and can we find adequate solutions? (3) Can we put together systems out of configurable as-is building blocks? (4) Can we bring effort and costs in relation to the similarity to an already existing application? (5) Can we trace the fulfillment of safety requirements? (6) Can we explain what the system does? (7) Can we generate enough trust into the systems - and how and by what means?

Of course, the solutions and approaches are not specific to robotics but to complex systems in general. In some sense, the domain of advanced service robotics is a moving target. As soon as a challenge in advanced service robotics is solved, that tends to be called automation subsequently. Thus, advanced service robotics just always pools all the cutting-edge challenges. That is why the focus of this chapter is not on all the positive examples of software systems for robotics, which made the implementation and deployment of all the successfully working robotic systems possible. The focus is on the challenge of Software Engineering for Robotics: advances here allow for opening up new markets for robotic systems and allow for entering new application domains by robots.

\subsection{The Power of Ecosystems and the Power of Separation of Roles}

Ecosystems are dynamic and co-evolving communities of diverse actors who create and capture new value through both collaboration and competition [7]. A distinctive characteristic of many ecosystems is that they form to achieve something that lies beyond the effective scope and capabilities of any individual actor (or even group of broadly similar actors). 
Ecosystems show benefits in coping with the ever-increasing complexity of many processes, products and services and the decrease of lot sizes [8]. More and more, services or products must be tailored to very specific requirements in order to make them a fit. This fit can best be achieved by individually selecting building blocks and putting them together according to the order to be fulfilled.

A huge variety is just not feasible within a single value chain. A value chain comes with a small number of stable partners. Business relationships just grow over time and some partners make it into preferred and established relationships. Instead, we now need to have access to a pool of thousands of partners that all are highly specialized experts in particular niches. This is because we need to select at any given time the then best fitting partner, even when this is going to be a partner for only a short period of time or for very low quantities of an asset. For the most part, supply chains of large businesses were not set up to deal with a world of thousands of partners, and they need to evolve into value networks (Fig. 3.3).

An ecosystem works differently. It requires partners to strictly focus on their unique expertise and allows them to become highly specialized. In an ecosystem, as purchaser, one depends on a huge pool of different offerings with finest differences mapped out. This allows purchasers to match the requirements of their customers
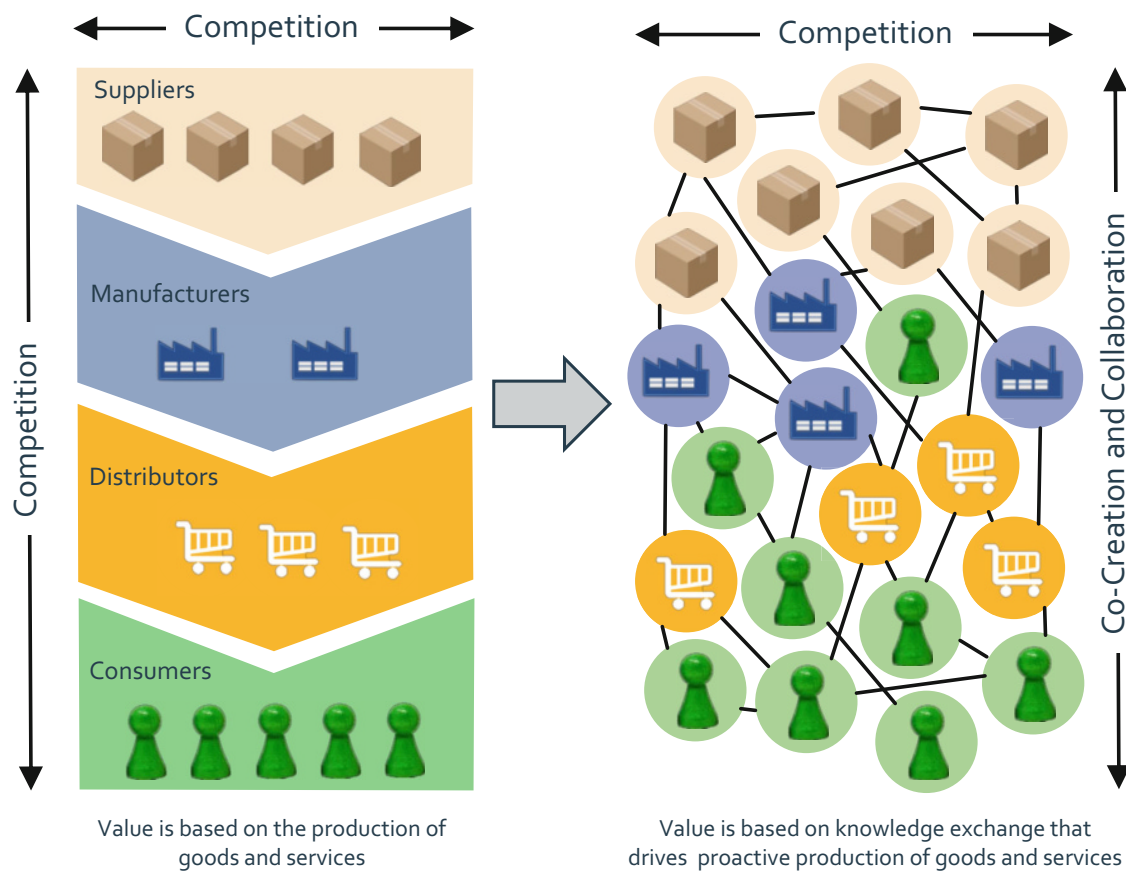

Value is based on knowledge exchange that drives proactive production of goods and services

Fig. 3.3 Linear supply chains evolve into complex, dynamic and connected value networks that build on co-creation, collaboration and access to a pool of thousands of partners within an ecosystem (Source: own illustration based on [8]). 
with products tailored to the orders. As a supplier, one depends on many different purchasers selecting the offered components or services in order to come up with high enough revenues. One just needs to be the best within one's narrow scope and then rely on others being the best in their niches. This is why an ecosystem as a whole is very agile in filling even the smallest niche and the smallest demand with dedicated offers. In an ecosystem, one does not depend on the small world of a particular value chain anymore. Instead, one reaches out to all the partners with opportunities to collaborate with all of them. With most of the partners, one collaborates only temporarily, but one always does so with enough partners to stay focused on one's particular expertise.

We call that way of splitting up work and distributing it across many different partners separation of roles. This is different to the way in which work is split up along a product-line approach [9] as separation of roles comes with a different granularity of management and with more responsibilities for a partner filling a role [10]. Of course, within our local scope, we can still run a product-line approach (see Chap. 1 for software product-line engineering).

Thus, the basic difference of a business ecosystem compared to a value-chain approach or compared to a product-line approach is its different way of managing the huge variety of partners (that all form their own organizational units) in order to better tackle a huge variety of tailored products. Instead of trying to manage all the required interactions and interfaces to cope with complexity, an ecosystem provides a set of mechanisms that allows thousands of partners to collaborate on demand and to do this under continually changing operating conditions. Management to ensure that the artifacts of the different partners fit together or that the partners smoothly interact in tightly coupled ways is replaced by structures and means that ensure those fit even under loose couplings between partners. A business ecosystem supports independent and locally managed work to end up with fits without extra management on top.

Figure 3.4 shows three different tiers that are typically found in a business ecosystem. The uppermost tier is driven by only a small number of partners, called the ecosystem drivers. At that level, all the basic structures and means that form sound foundations for the other tiers are provided. Tier 1 can also

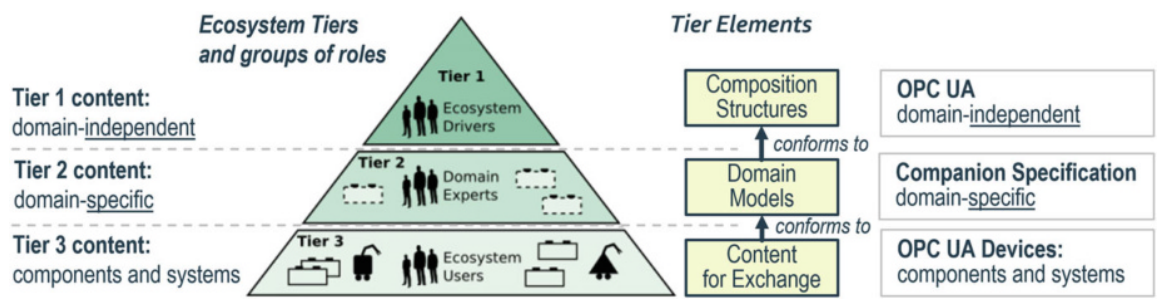

Fig. 3.4 Three tiers typical for any business ecosystem. Tier 3 is conforming to Tier 2 and Tier 2 is conforming to Tier 1 . While there are only few ecosystem drivers forming Tier 1 , there are already more domain experts at Tier 2 and finally all the ecosystem users are at Tier 3 . 
be called foundations. The middle tier is driven by domain experts that use the Tier 1 structures to come up with domain specifics. The middle tier is completely conforming to the uppermost tier. The separation between Tier 2 and Tier 1 is extremely important as Tier 2 allows to shrink the size of the domain under consideration such that agreements on structures become feasible. Tier 2 allows us to have many different domains in parallel and without conflicts in consistency, even coexisting and competing approaches for the same aspect. For example, the navigation domain and the vision domain might differ in the representation of coordinate systems. There might be different vocabularies for specifying trajectories all consistent in themselves. Of course, fragmentation without reason is not good, but Tier 2 allows for processes to resize scopes of domains and to sort out competing approaches where they are just different without any reason. As any Tier 2 domain is conforming to Tier 1 , one can build links between different Tier 2 domains via Tier 1 . Tier 2 is typically filled by domain-specific working groups. Tier 2 can also be called definitions. Finally, Tier 3 comprises all the concrete products and services that conform to at least one of the domains at Tier 2. As purchaser, one knows beforehand which parts can fit together. As supplier, one knows to what kind of structures one needs to adhere to so that the provided parts fit to others. Tier 3 can also be called implementations.

This is exactly the same structure that is also followed by $O P C U A$ in the context of industry 4.0 [11]. OPC UA provides the basic means for information models and accessing them (i.e. Tier 1). However, that still is not enough to make different industry 4.0 devices interoperable. Interoperable devices must use the same information models, that is, they must apply the generic OPC UA concepts in the same way. Thus, OPC UA introduces the so-called companion specifications (Tier 2) [12]. Finally, Tier 3 comprises all the conformant devices.

\subsection{The Power of Composition}

Complexity can be mastered by splitting up a complex problem into smaller ones, solve those and finally put these solutions together to form a solution for the original problem. Of course, that proved to be a reasonable approach in many engineering disciplines as we then can assign the work to different entities, let it be persons, teams, departments or even contracted other companies. This lets us do the work faster as we can get it done by a larger workforce. We can get it done concurrently and we can also identify whether there are already solutions for subproblems that we might want to reuse.

However, just splitting up the problem or splitting up the work is not enough. It needs to be done such that the subparts ultimately fit together and that they properly form the intended solution. Different approaches address exactly that challenge in significantly different ways [13].

A whole set of approaches is based on integration. The outcome of integration is an amalgamation of the previously separated subparts. It is quite difficult (and 
typically not foreseen) to again split apart the outcome afterward or even modify it. As everything is optimized for a set of preplanned configurations, any changes or variations interfere with the highly optimized setup that works like a clockwork. Basically, there is a huge management effort needed in keeping all the interactions between teams, and interfaces of parts and the work in general (i) up and running, (ii) in sync and (iii) orchestrated, so that at any point in time everything fits smoothly. Product-line approaches to face variations work well only within the same organization $[9,10]$ and as long as it is about foreseen variations of the same product for which the product line got already prepared. As soon as we need to cross organizational boundaries (i.e. when we are in need of managing resources not under our control, like other teams, other departments or external contractors), integration-based processes reach a level of complexity that cannot be managed anymore.

Another set of approaches is based on composition. It is the activity of selecting building blocks (modules, components, subsystems, etc.) as they are and putting them together. Composability is the ability to combine building blocks. Compositionality refers to the ability to understand a composite system by understanding its building blocks and how they are combined. Modularity is the degree to which a system's building blocks may be separated and recombined. Modularity comes with the challenge of designing building blocks with well-defined interfaces that can be used in a variety of contexts. Composition is one approach to achieve modularity. In contrast to an integrated system, a composed system can again be split apart into its building blocks. Thus, modifications and adaptations are possible requiring only adequate effort. Adequate means that effort and costs are in balance with the achieved result. That is exactly how we build complex Lego models out of given Lego bricks. Of course, as long as only regular bricks are used, many contours and shapes can only be approximated. Nevertheless, there is still room for most specialized bricks. These fit as long as they adhere to the standardized knobs. However, specialized bricks come with a price to pay in terms of extra costs, extra effort and less reuse in other contexts which does not pay off in case a solution based on standard bricks is adequate.

For example, resource shares and reservation-based mechanisms are composable. As long as there are resource shares available, these can be claimed without interfering with already assigned shares. However, priorities are not composable. Each new asset might require reassignments of priorities (see, e.g. priority assignment in rate monotonic scheduling).

To summarize, composition is a very powerful concept to build a huge range of diverse and modifiable systems in an economically feasible way, even down to lot-size one, as one does not start from scratch over and over again. Instead, one just reuses (compositions of) commodity building blocks as far as possible. This amortizes costs at a different level of granularity. It is not anymore by huge quantities of the same and difficult to modify finished product. It is at the level of huge quantities of commodity building blocks that become part of many different and diverse finished products. 


\subsection{Aiming for a Software Business Ecosystem for Robotics}

Robotic systems need to become more flexible, more adaptable and still affordable even when it comes down to lot-size one for a particular robot. We select, combine, adjust and exploit those already available software engineering approaches that help us best in matching those needs. The approach is not limited to robotics.

A business ecosystem provides a lot of benefits in addressing the above challenges. In order to ramp up a business ecosystem for robotic software systems, one first needs to identify what structures need to be defined at which tier. One also has to answer the question of how those structures are presented to participants of an ecosystem. For them, it needs to be as simple as possible to adhere to the defined and agreed key structures and to apply those structures properly and in the intended way. This is of paramount importance in enforcing the principles of separation of roles and of composition as these form the pillars of a working and fruitful business ecosystem.

Figure 3.5 gives an overview of some core concepts. First of all, it shows different roles, such as a component developer, a system builder but also a safety engineer, a domain expert, a system architect and a behavior developer. For example, a component developer selects functional libraries from marketplaces of the ecosystem and uses them within software components. A system architect translates the requirements of a domain expert into a system design. A system builder selects those software components that fit best to a design and composes them to form the system. A behavior developer models action plots to achieve a certain task but in a way that is independent from a specific robotic system. Rather, a particular action plot needs to be executable with all robots that have the required capabilities. A safety engineer translates domain-specific safety requirements into a set of constraints that are checked during the development as well as during the operation phase. There are many more roles, but it is important to understand that they all work concurrently and independently from each other and that there can be many partners for the same role at any time.

As shown in Fig. 3.5, the structures of all assets, whether these are functional libraries, software components or action plots, are represented via blocks with ports, where ports are linked by connectors. A block separates the internals from the externals. The only way to interact with a block is via its ports. Blocks can be nested as is the case, for example, of a software component that comprises functional libraries that are also blocks with ports. As soon as the experts of a particular domain agree on the kind of blocks, ports and connectors for the different assets of their domain (of course, structure and behavior), one can ensure that conforming assets finally fit together in the intended way.

Figure 3.5 also shows that each block comes with a data sheet. This proved to be an extremely successful mechanism to decouple suppliers and purchasers in a business ecosystem. A data sheet describes an outside view of an asset, including its foreseen variation points. A data sheet includes internals only as far as we need to know them for using the asset and for predicting its fit (behavior, structure) for our 


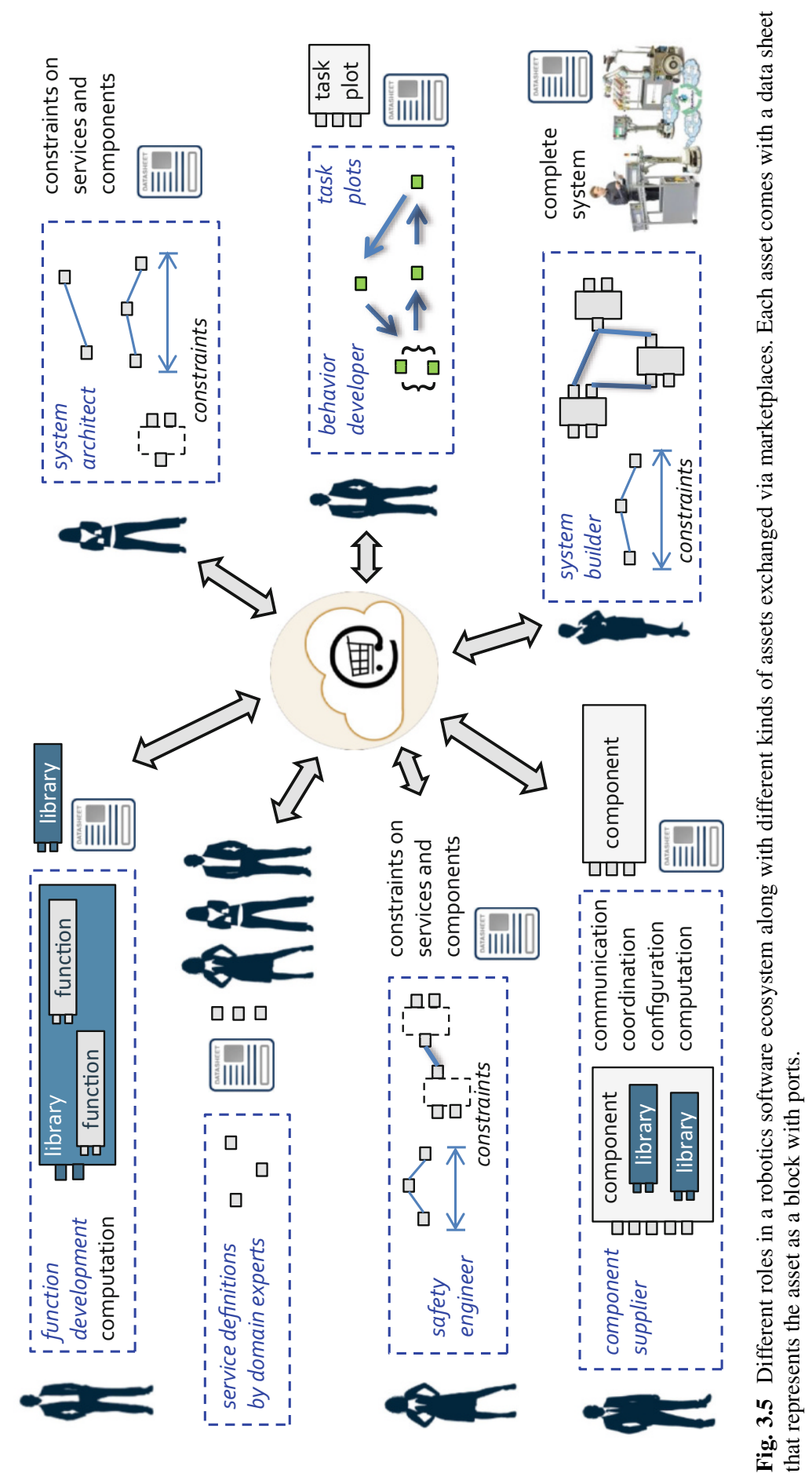


context. Data sheets are, by purpose, not rich enough for synthesizing the artifact. They are an abstraction of the asset and they are not in conflict with our needs in protecting our intellectual property. Data sheets are models. A data sheet is the minimum information we need to provide to users about our asset. The asset cannot be operated, and thus would be useless, if one does not know what to provide to its ports and how to set its variation points.

\subsection{The Role of Model-Driven Software Engineering and of Data Sheets}

It is important to understand what kind of roles we foresee for models and for model-driven software engineering in a business ecosystem for robotics [14]. An essential part of software engineering is managing interfaces between software assets. Despite the different levels of granularity of software artifacts, one ends up with different types of interfaces such as programming interfaces for libraries, service-oriented ports of software components and many others. These interfaces can all be represented by blocks, ports and connectors.

Models are considered as the most adequate way to explicate and link all the required and agreed structures in a consistent way. Models are not bound to a specific implementational technology. The represented insights are decoupled from the pace of new technologies. Models allow for early binding of semantics and for late binding of technology (in source-code-driven approaches, it often is exactly the other way around). Models form the baseline for role-specific model-driven tools that enforce the agreed structures and thus ensure that outcomes of independent work fit into the ecosystem.

Model-driven software engineering and associated model-driven tools are strong (1) in making structures with their semantics accessible and (2) in enforcing those structures with their semantics. In a robotics software business ecosystem, all those structures that implement the principles of separation of roles and of composition should be managed by a model-driven approach. Compliance to relevant structures and to their semantics is not anymore achieved by the developer's discipline. Instead, this compliance is ensured by the model-driven tools that generate (or just retrieve) the related source code for the asset's interfaces. Pregiven reference implementations for interfaces are a feasible way to provide their semantics (meaning and behavior of the structures). Model-driven tools relieve users from a huge cognitive load and from a huge responsibility while still giving all the freedom beyond the enforced structures.

We do not advocate fully replacing source code by models. Coding is to some extent modeling as well and models in the form of source code are even executable. Instead, we understand models as abstractions of artifacts, then called a data sheet. The more is expressed in the data sheet, the more details can be taken into account 
for prediction, analysis, etc. and the less there is a need to actually build all the variants in a costly way just to identify-by real-world testing - the wanted variant.

A model in the form of a data sheet does not cover everything to the finest detail, and it does not allow the synthesis of the represented artifact. Of course, the better a model can represent further properties of an artifact, the richer we can make the data sheet. Indeed, this can be a reasonable migration path to full-blown models and a full-blown model-driven approach. If it becomes both beneficial and feasible to avoid coding at all, then there might be a shift toward fully model-driven software engineering approaches. However, we might still end up with considering coding as the more efficient way of expressing some of the details. The reason is quite simple: software models are not just a level of indirection, they are an abstraction. A software model does not explicate all the complex details of its target platforms. Anyway, it is about a beneficial coexistence and about consistent links between different abstraction levels.

Figure 3.6 shows a fully model-driven approach where all the different models are composed to the final system (see (1). However, pure modeling without means to get models grounded in the real world is not a solution in robotics as robots finally need to act in the real world. Indeed, a model-driven approach in robotics makes sense only when there are no gaps between models and their grounding in real-world assets. After modeling is complete, one needs mechanisms to transform the models into an executable form. Up to now, such mechanisms exist only in a few domains. For example, $3 D$ printing is such a domain as a $3 \mathrm{D}$ printer is such a generator (see (2)). As result, we get the real asset and a data sheet (see (3). The data sheet is an excerpt of the model used to generate the artifact and contains only information that the user needs to know for using the asset. Unfortunately, such a generator is still not there for complex software systems.

Figure 3.7 shows the approach we are favouring for a model-driven software approach in robotics. As a data sheet is an abstraction of a real asset, there is no data sheet without a related real asset. We can already compose a system at the model level by composing data sheets. This allows us to already check those system-level properties that are covered by the data sheet abstractions of the real assets (see also
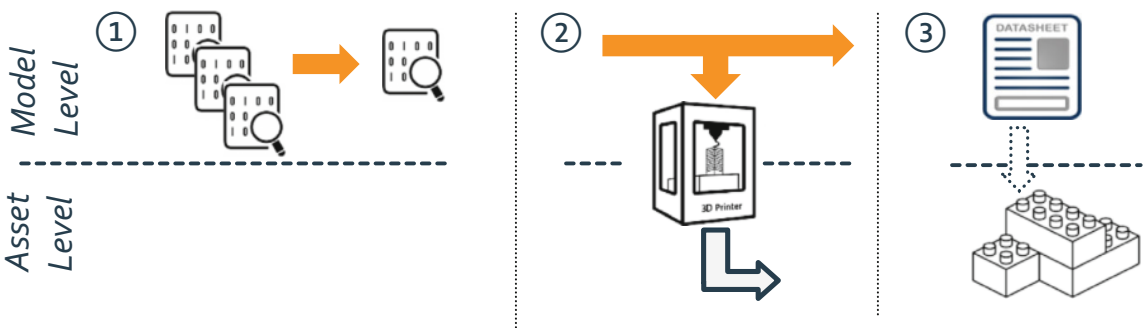

Fig. 3.6 A fully model-driven approach where we first model everything (1) before we finally use a generator (2) to get out of the model the real-world asset and an excerpt forming the data sheet (3) 

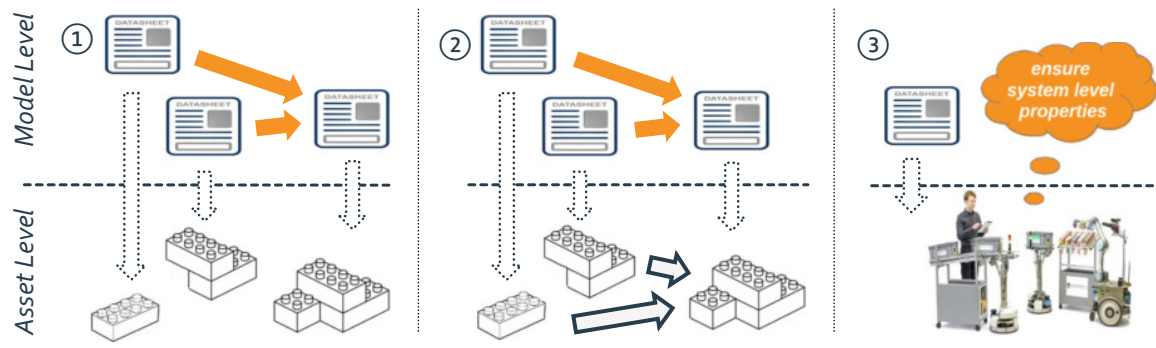

Fig. 3.7 Data sheets are models that are abstractions of real assets. A data sheet describes an outside view of an asset and it is grounded via the real asset.

Chap. 4 on testing, Chap. 7 on verifiable autonomy and in particular Chap. 8 on verification). For example, we can already perform various what-if analyses and we can trade off configurations of the variation points of the assets (see (1). This can all be done prior to buying any of the assets. Once we are fine with the predicted outcome, we can go for the according real assets (see (2)). The transformation from the model level to the real-world level is by picking up the related assets and putting them together in the same way as we did at the data sheet level. This gives us a system with properties consistent to the predicted ones (see (3)). Of course, all those properties of the system that are not covered by the data sheets can only be checked via the real assets.

This chapter is organized as follows. Section 2 describes the arrangement of software technologies such that a robotics software business ecosystem can get shaped. Section 3 goes in-depth into selected details of Tier 1 as this tier is decisive for the overall ecosystem approach. Section 4 gives selected insights into Tier 3 and into a world of model-driven tools from a user perspective as most ecosystem participants will operate at Tier 3. By that user-centric view, the role of Tier 2 and its links to Tier 3 and Tier 1 get clear as well. Section 5 relates the presented work to state of the art and concludes.

\section{Structures for a Robotics Software Business Ecosystem}

Separation of concerns is an important design principle to handle complex interdependent systems [15]. An example is the Internet protocol stack with its different well-defined layers. A good design defines abstraction layers where each layer can exist without the layers above it, but requires the layers below it to work. Otherwise, different layers just form levels of indirection. By definition, a layer cannot be defined on its own, since its semantics is the relationship between its artifacts and those of the neighbouring layers. The exercise to obtain well-defined relationships is a tough one. Of course, transitions between layers can be fluent and individual layers may also be split horizontally. There can also be implementations that just 


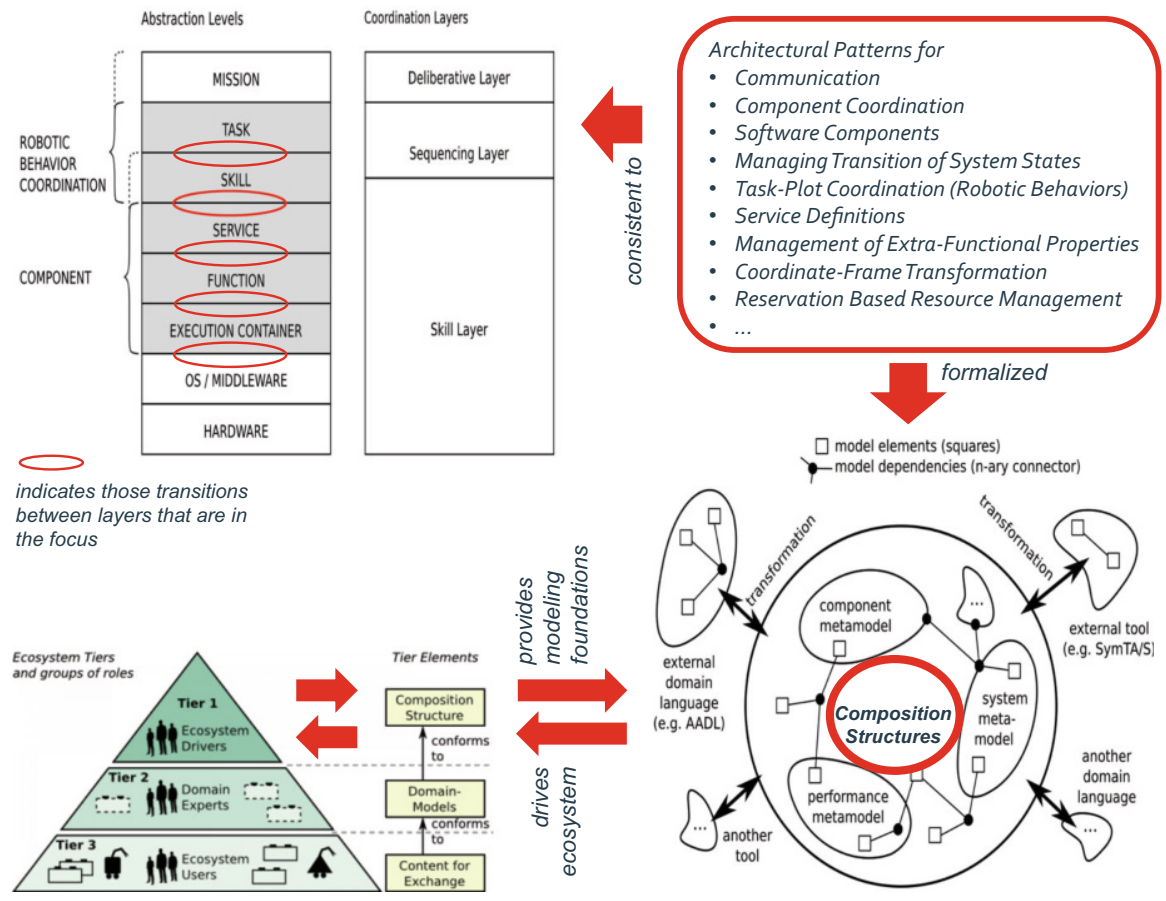

Fig. 3.8 Best practices and lessons learned are described in the form of architectural patterns. Experts translate architectural patterns into formal models using block-port-connector meta-models. Formalized and consistently arranged architectural patterns form the composition structures. Composition structures form the ecosystem foundations.

combine several adjacent layers into one, which means losing flexibility. However, it is not good practice at all to define relationships between non-adjacent layers.

Figure 3.8 shows widely agreed layers for robotic systems. The lowest layer is hardware, followed by the operating system/middleware. The execution container provides access to resources in a way independent of the implementational technology of the layer below. The function layer comprises the implementations of the algorithms. A service performs work based on contract and with its own resources and thereto interacts with other services. A skill arranges different services such that they become a particular robot capability. It translates between a generic name for a capability like move (from above) and configurations and parameterizations specific to the used services (downward). The task layer represents what steps and how these need to be executed in order to accomplish a particular job. Tasks are independent from a particular robot but can be executed with a particular robot only if that robot possesses matching skills. Finally, the mission layer represents the purpose of the robotic system.

The challenge is to define the relationships between the different layers. We need to find those patterns and structures that form the sweet spot between freedom of 
choice and freedom from choice [16, 17]. We need to support as much freedom as possible to not block progress and to address specific requirements in the most flexible way. On the other hand, we need to define at least those structures that ensure composability and separation of roles and that overcome fragmentation. The latter is about just different alternatives for the same concept but without any specific reason and without providing added value.

Obviously, it is not as simple as separating the overall robotic system along both the above abstraction layers and the different concerns such as computation, communication, coordination and configuration. This would end up in a granularity of assets that neither fits to a natural role in a robotics ecosystem nor forms marketable entities. In contrast, a software component is a good example for a reasonable building block as it is a marketable asset that fits to the well-understood role of a component developer. Of course, a software component comprises aspects of all the above concerns. Nevertheless, a well-defined software component model jeopardizes neither abstraction layers nor separation of concerns.

We introduce the notion of an architectural pattern to describe a particular recurring design [18]. An architectural pattern is a textual description comprising a specific design context, a recurring design problem and a well-proven solution. In our case, architectural patterns are driven by two fundamental objectives, that is, (1) to facilitate building systems by composition and (2) to support separation of roles. Architectural patterns allow us to compile knowledge and best practices from a community.

Via the description of the specific design context, it gets clear whether the pattern belongs to Tier 1 of an ecosystem, whether it is specific to a particular domain and thus belongs to Tier 2, or whether it is specific just to particular assets and thus belongs to Tier 3. Architectural patterns already allow in their textual form to identify competing approaches and overlap in scope. We can then initiate discussions, clarifications, classifications and decisions to ensure overall consistency across the different tiers. Afterward, all that can be formalized by using the fundamental modeling means as provided by Tier 1 .

\section{1 (Meta-)Models and Tiers}

The concepts of conform-to, models, meta-models and tiers are key in understanding how an ecosystem gets organized. All the concepts at Tier 1 are prescriptive for Tier 1 and Tier 2. All the concepts at Tier 2 are conforming to the concepts at Tier 1 and they are prescriptive for Tier 2 and Tier 3 . All the concepts at Tier 3 are prescriptive for Tier 3 and they are conforming to Tier 2.

At a particular tier, one can only operate in a way that is consistent with the scope of the already given prescriptive concepts. If there is already a prescriptive concept at a tier, one cannot go for an alternative one at a lower tier. The only option is to add at the tier comprising the prescriptive concept an alternative concept that is placed next to the already existing one. The alternative concept nevertheless still needs to 
be consistent with the concepts at this tier and with the prescriptive ones from the above tier. The more alternatives get introduced, the more we again end up with fragmentation that destroys composition. This is why it is so important to come up with very well thought-out concepts and place them at the proper tier.

For example, the key elements of the structure and the behavior of a software component are defined at Tier 1. This means that all the software components within that ecosystem adhere to that structure. The prescriptive parts of a software component are, for example, the basic states of its lifecycle automaton, the structure and behavior of its ports (such as a request-response or a publish-subscribe interaction), its behavior interface for its configuration and many more. The model of these key elements that constitute a well-formed software component is part of Tier 1. At Tier 2, a software component can still be tailored to a specific domain, for example, by the substates of the lifecycle automaton, the concrete data structures exchanged via the ports, etc. The prescriptive models of Tier 1 are used at Tier 2 to come up with a concrete set of domain-specific ports that are conforming in structure and behavior to the prescribed elements. The concrete set of domain-specific ports, which is defined at Tier 2, is again a set of models. This set of models is conforming to the related Tier 1 model and that Tier 1 model is a meta-model for it. Finally, a concrete software component is modelled at Tier 3, using the Tier 1 software component model as meta-model and the domain-specific refinements of Tier 2 as another consistent meta-model.

Tier 1 contains all structures that are relevant to shaping an ecosystem and that are independent from a specific domain. Nevertheless, these still provide all the hooks to get tailored at Tier 2 to domain-specific needs where necessary. For example, how to represent skills is defined at Tier 1, but the very concrete and domain-specific names of capabilities are defined at Tier 2. The structure and behavior of all the elements of a software component including the structure and behavior of its ports are fully defined at Tier 1, but the concrete set of instantiable domain-specific service ports are defined at Tier 2 .

\subsection{Tier 1: Foundations}

This tier comprises all the sound foundations for expressing ecosystem structures. Tier 1 itself is organized into three different layers as shown in Fig. 3.9. The structures in Tier 1 are provided and driven by experts. The overall community compiles its body of knowledge via architectural patterns. Groups of experts moderate the consistent arrangement of the architectural patterns and then formalize them to become part of the bottom layer of Tier 1. As component developer, system builder, etc., one does not get directly in touch with those structures as one uses rolespecific tools. The fully detailed description of the following concepts is available via the RobMoSys Wiki [19]. The Wiki is an effort of the robotics community that is driven and moderated by the EU H2020 RobMoSys project. It aims to explicate the body of knowledge of the robotics community for the purpose of ramping up a 


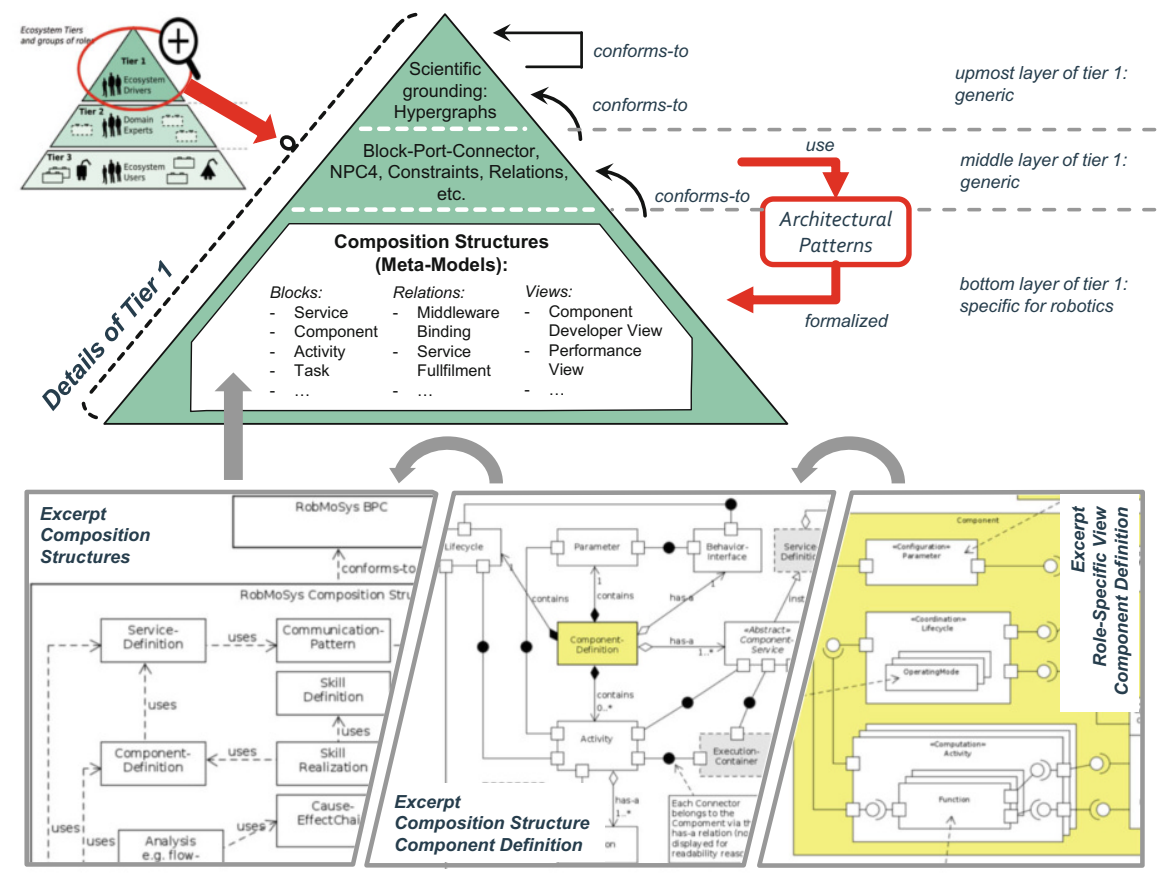

Fig. 3.9 A modeling view clusters related modeling concerns in one view. A view establishes the link between primitives in composition structures and ecosystem roles. Views enable roles to focus on their responsibility and expertise.

digital industrial platform for robotics. The euRobotics AISBL with its topic groups already adopted the role of stewardship for the robotics body of knowledge in order to ensure sustainability. Stewardship is about the responsibility to moderate, shepherd and safeguard the community's body of knowledge.

The uppermost layer provides a hierarchical hypergraph model as scientific grounding [20], [21, hypergraph-er]. A hierarchical (property) hypergraph [22, 23] is the modern, higher-order version of the entity-relationship model. In short, hierarchical means that every edge and every node can be a full graph in itself. In other words, any relation can be considered an entity in itself and can hence be used as an argument in another, higher-order relationship. Hypergraph means that every edge can join any number of nodes, that is, it is an n-ary hyperedge. Property meta-data means that every node and every edge in the graph has a property data structure attached to it (at least, a unique identifier and a meta-model identifier).

The middle layer contains the entity-relationship model, which is a specialization of the hypergraph model and that conforms-to a hypergraph. It comprises the concepts of an entity, a relation and a property and the basic set of standard relations is-a, instance-of, conforms-to and constraints. Their semantics is not specific to 


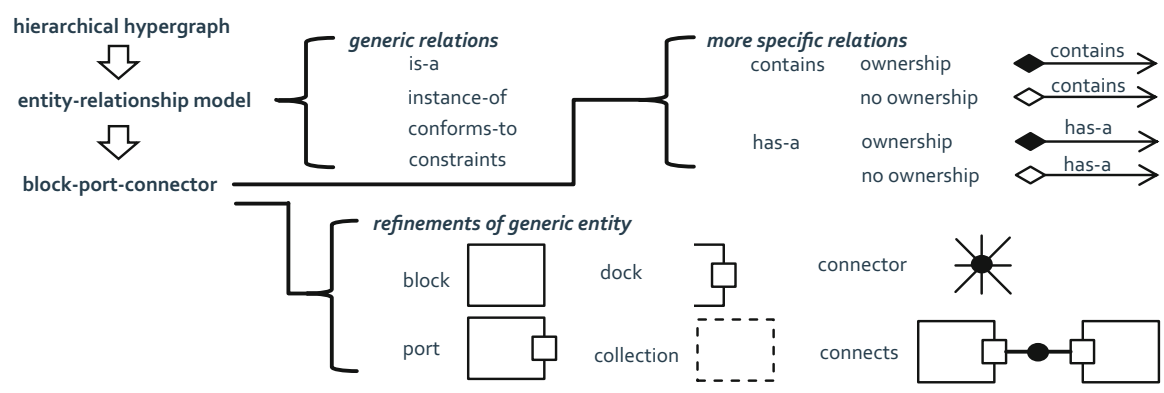

Fig. 3.10 Some selected entities and relations of the RobMoSys modeling foundations (see [21, principles:block-port-connector] for full details).

robotics, but is given by just typical common-sense descriptions. These are basically backed by generic modeling foundations such as mereology and topology.

This allows us to introduce the block-port-connector model, which is a specialization of the more abstract hypergraph model and of the entity-relationship model. It comes with two additional relations, namely, contains and has-a. The generic entity is refined into block, port, dock, connector and collection, and the generic relation is refined into connects. All these come with a description of their semantics (Fig. 3.10 and [21, principles:block-port-connector] for details).

We now have all the means to express structures and their relations by a blockport-connector model. Please note that while blocks and ports are semantically different, the structure of a port is represented as well by blocks. The kind of presentation is specific to a particular view, its purpose and its level of abstraction.

The bottom layer comprises the composition structures. The entry point for their detailed description is again the RobMoSys Wiki [21, composition-structures:start]. These are formed by the consistently arranged architectural patterns that are then formalized using the elements that are provided by the middle layer of this tier. This gives a set of meta-models [21, metamodels:start] that define the various elements and their relations. An excerpt of the composition structures is shown in the lower left-hand side of Fig. 3.9. The lower middle part of Fig. 3.9 shows a snippet of the meta-model of a component [21, metamodels:component] that comprises all the elements with all the internal and all the external ports that make the structure of a component and its links. In contrast thereto, the snippet on the lower right-hand side shows only those parts of the component meta-model that are relevant to the role of a component developer. A role-specific view is a collection out of those meta-model elements that are relevant for guiding in a consistent way the work of that role.

As we use blocks, ports and connectors to organize structure, behavioral aspects also get structured and separated along blocks, ports and connectors. The advantage now is that for describing the behavior of a block, it is sufficient to describe for all its ports (1) their behavior (just the behavior of the port as it is visible from outside the block) and (2) which input port influences which output port (connections inside a block and related transfer functions). This is fully in line with the way of thinking 
of the above-described data sheet approach. As long as not all relevant properties can be made available in a formalized way, and as long as one cannot deal yet with all the details due to overall complexity, one can already now offer a migration path toward this end by stepwise making data sheets richer and richer. A valuable approach that is already feasible now are compliance checks via running tests. Of course, there needs to be a mutual understanding and agreement about significance and coverage of the set of tests for properties of interest. In the same line are tests that run an artifact against a reference artifact to check whether there are deviations in properties defined as relevant. Another approach is to run artifacts in simulation to see whether the produced behavior is in line with the expectations (at least for the properties considered as relevant).

All the composition structures conform to the middle layer elements and, thus, either just use that semantics or they come with a consistent refinement that is further narrowing that semantics. Again, the semantics is specified either (1) by references to outside documentation, or (2) by referring to related reference implementations, or (3) by transformations into other representations that already come with a semantics. Of course, at least one mechanism, and sometimes even all three, is given for all the specific elements that are introduced in modeling the composition structures.

\subsection{Model-Driven Tools to Access and Use Tier 1 Structures}

The modeling mechanisms of Tier 1 are independent from any technology. Nevertheless, making them accessible and usable via model-driven tools has significant advantages in addressing what is called the semantic gap. Often, there are only textual descriptions of the semantics. Without model-driven tools, one depends on the users to properly interpret that semantics and to come up with a correct use in their particular context. There is a high risk of misinterpretations if different users do that individually. In a model-driven approach, highly specialized experts do this once and transform their reasoning and interpretation just into additional structural constraints. Pitfalls on the user side can be sorted out as some assets then fit only in the intended way or get directly linked to a trustworthy implementation providing the intended semantics without any chance to circumvent it. Structural constraints are easy to implement even with model-driven tools of limited expressiveness.

As illustrated in Fig. 3.11, the modeling mechanisms are represented in different ways in different model-driven tools. The modeling elements in the model-driven tools consistently refer back to the full set of Tier 1 structures by the unique identifiers that are used with all modeling elements. Unique identifiers are used, in particular, for elements of instances, elements of models and elements of metamodels.

Examples of specific technologies in model-driven tools are Eclipse Ecore and $U M L$ profiles. Although neither has enough expressiveness to directly represent the structures of Tier 1, one can still implement with them role-specific views. A role- 


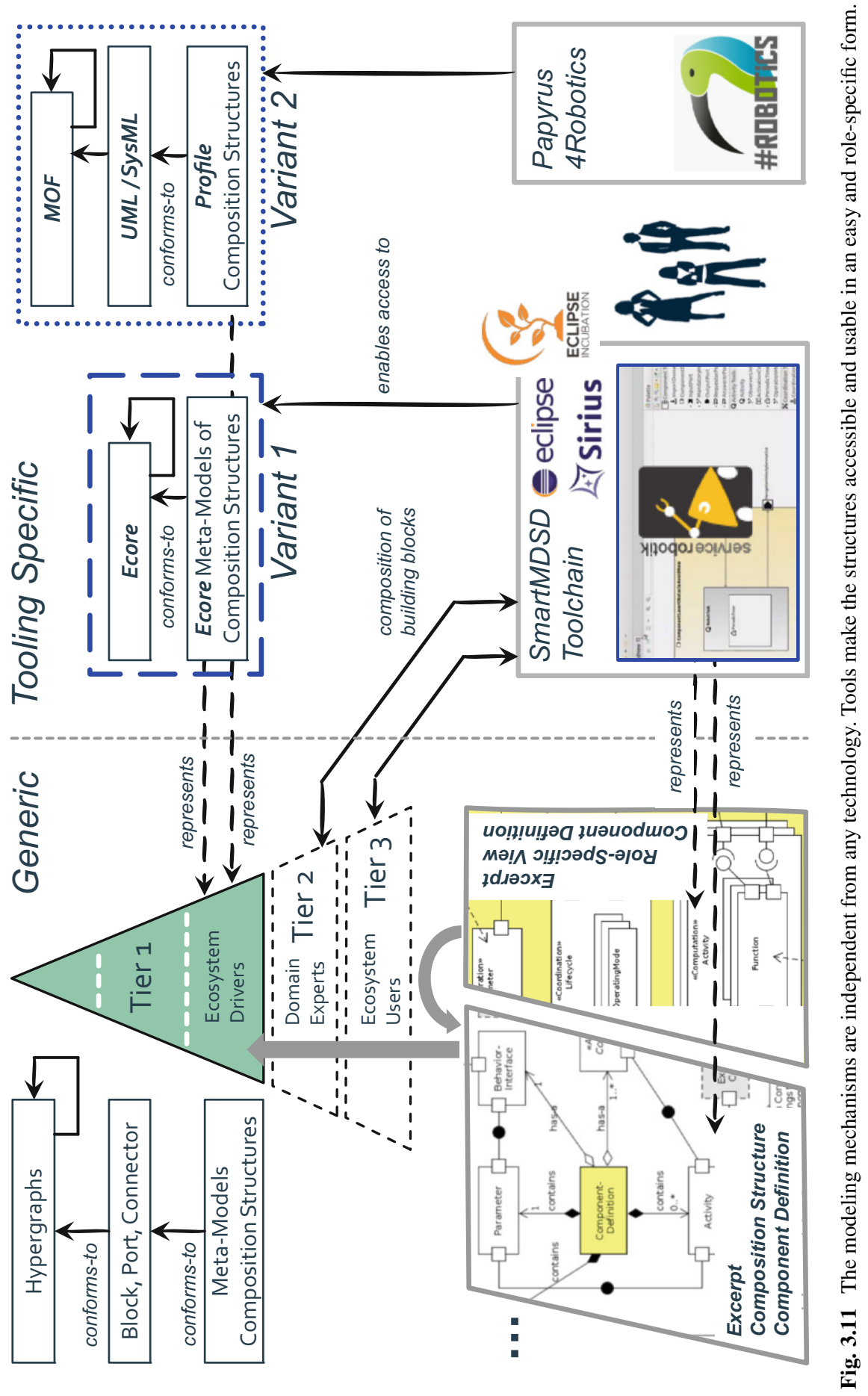


specific view allows a particular person in its role to do its work within its context in a consistent way. Missing expressiveness can be circumvented much easier in the narrow scope of a particular view as measures can be specific. For example, an often-occurring challenge is to transform models about what is not allowed into proper mechanisms of model-driven tools. An approach often used for this is to structure the presentation of the view (guiding the user through particular steps) and to run checks for well-formedness before one can proceed with the next step.

\subsection{Tier 2: Definitions}

Tier 2 structures all the domains that are part of an ecosystem. A particular domain, such as vision, navigation, manipulation, etc., is shaped by the experts of that domain. Again, it is a community effort to structure a particular domain by creating domain-specific models. As a domain is limited in scope, it is much easier to come up with an agreement about, for example, particular vocabularies and data structures. At Tier 1, one would need to aim for an all-encompassing approach which several times already proved to be just infeasible (as evidenced by all the failed approaches to come up with a single ontology for a domain such as robotics). However, as all the domain-specific models in Tier 2 conform to Tier 1, these different models can be linked to each other in a consistent way.

By the way, that is exactly the role of meta-models. It is important to understand that the concept of a meta-model is a relative one. A meta-model is just a model as well. It becomes a meta-model only with respect to other models that conform to it.

As Tier 1 serves as meta-model for Tier 2, all models at Tier 2 conform to the models at Tier 1 . Models at Tier 2 bind flexibility and narrow down semantics, but they are all consistent with the models in Tier 1 . Of course, there can be different and coexisting domains, all with their own models. Although these are all consistent within their domain and although they all conform to Tier 1, concepts often cannot be easily linked one-to-one across domains. However, such links between domainspecific models are always available via the path through Tier 1.

Obviously, Tier 2 needs to be defined within the structures and semantics given by the bottom layer of Tier 1 . In order to fulfill the relation conform-to, one can neither just pick out structures and ignore their semantics, nor can one just arbitrarily pick out elements from the top layer and the middle layer of Tier 1 as these layers do not form the interface to Tier 2. One cannot just go upward until it gets generic enough to then extend from there on. Of course, doing so would completely break the sound conceptual approach of layering abstraction levels and having them only interact with adjacent layers. It would break all the foundations that enable composition and that would again result in fragmentation.

If we cannot live with the framing of the adjacent superordinated layer, we cannot just put a different semantics next to it and ignore what already has been agreed. Instead, in case there are very good reasons why adjustments or extensions are needed at a particular layer, then we should just submit a convincing proposal 
that has a chance to get support from the community or expert group. As already mentioned before, that is why there is such a high responsibility in coming up with proper structures that match the sweet spot between freedom of choice and freedom from choice.

Very often, the above is misunderstood and it takes some effort explaining why it is not sufficient to just go for the structural approach of blocks, ports and connectors. Blocks, ports and connectors become of use only when one respects their concrete designs with semantics. By the way, that is exactly the challenge of applying SysML. It fits easily nearly everywhere in systems engineering as it basically just refers to blocks, ports and connectors. However, it gets useful only when there is another level of detailing to provide a consistent meaning and grounding for the kinds of blocks, ports and connectors in a particular domain.

\subsection{Tier 3: Implementations}

Tier 3 comprises all the content available in the ecosystem. In robotics, such content is libraries, software components, solution templates representing architectures as well as task plots that convey process knowledge independent of a particular robotic system, and many other artifacts. Tier 3 uses the domain-specific structures from Tier 2 in order to produce content to supply to the ecosystem and thereby using content from the ecosystem. For example, an expert in localization will adhere to the services defined for the navigation domain in building a software component for localization as it then fits to others conforming as well to the agreements for the navigation domain.

\subsection{Coverage and Conformance}

An ecosystem is viable and sustainable only when it finds the right balance between rigidity and plasticity. The further up a concept is in the hierarchy of the ecosystem structures, the more impact on the ecosystem comes with any modification of that concept and the better reasons have to be brought up for doing so. Thus, proposing an alternative to what is already defined (without having a real benefit, a real need or high enough added value) will hopefully not find enough supporters as this just fosters fragmentation. It is much simpler to make modifications at a lower tier of an ecosystem as the scope of the impact is much smaller. However, the lower a concept is in the hierarchy, the more constraints for new concepts come from the superordinated structures to which conformance is mandatory. In principle, there is always the option to put something next to what already exists (e.g. open up a new domain at Tier 2) as long as it is conforming to superordinated structures. This is an important means to take up new proposals and then perhaps let them even replace already existing structures. 
Basically, the balance between rigidity and plasticity is dependent on the processes that manage the ecosystem. Of course, the three tiers already come with well-defined responsibilities (ecosystem drivers at Tier 1, domain experts at Tier 2 and all the ecosystem participants in Tier 3). This makes it easy to identify and then approach the according representatives whenever needed. However, that alone is not sufficient. In the ideal case, an ecosystem is managed in the form of a meritocracy for which the following key processes are decisive.

First, there need to be processes for the vertical interaction (i.e. between stakeholders at different tiers) and for the horizontal interaction (i.e. between stakeholders in different parts within the same tier). For example, there might be a concept that evolved within a particular domain and that might be of fundamental use for other domains as well. In such a case, one should discuss whether to move that concept up in the hierarchy. In case one detects coexisting approaches at lower tiers for the same concerns, one should stipulate a process to overcome this accidental fragmentation.

Second, there need to be processes to assign coverage and conformance labels to assets in the ecosystem. That enables us to explicate for any asset its link into the ecosystem. Assets include concepts, models, software components, tools and everything else of relevance in the ecosystem. This tremendously simplifies navigating through the ecosystem and selection of assets according to needs. Of course, there can be coexistence of assets that come with only partial conformance (relevant for uptake of new ideas in early stages or for establishing first links into neighbouring domains), but this always is explicated.

Basically, the outcome of processes to tackle aspects of coverage and of conformance are agreements (1) on content and format of data sheets for all kinds of assets in the ecosystem and (2) on procedures to state the properties in the data sheets. Of course, coverage of particular aspects with their particular degrees of conformance can be subsumed under dedicated labels. Some labels can even come with certification bodies.

\section{Details of Selected Concepts at Tier 1}

This section explains details of some selected concepts at Tier 1 . These come with concrete structures and behaviors that are not specific to any domain in robotics. Exhaustive descriptions with lots of references to further documents are maintained via the RobMoSys Wiki [19].

A model at Tier 1 is called a definition meta-model when it defines the means for Tier 2 domain experts to model domain-specific aspects. It is called realization meta-model when it defines the means for Tier 3 ecosystem participants to model (and build) concrete assets. Otherwise, a model comes without any specific label. Of course, a model at Tier 1 directly serves as meta-model for Tier 3 in case there are no further refining models down to Tier 3. 


\subsection{The Software Component Model}

A software component $[24,25]$ is one concrete example of a block with ports that needs to be specified (structures and behaviors) at the lowest level of Tier 1. A software component combines aspects of different concerns, namely, computation, communication, coordination and configuration. It comprises the abstraction levels ranging from the execution container to the skill interface but without diluting their layering and their interfaces.

Figure 3.12 shows the structure of a software component, that is, its elements and their relations. Of course, these elements all come with well-defined behavior, and the behavior of the software component model is defined by the behavior of these elements in the context of the interaction given by the explicated relations. Thus, it would be a complete misunderstanding if one conforms only to that structure but ignores the related specified behavior.

For example, the Lifecycle of a component is represented as a block with ports. It interacts with the Activity as an activity runs only in particular states. It interacts

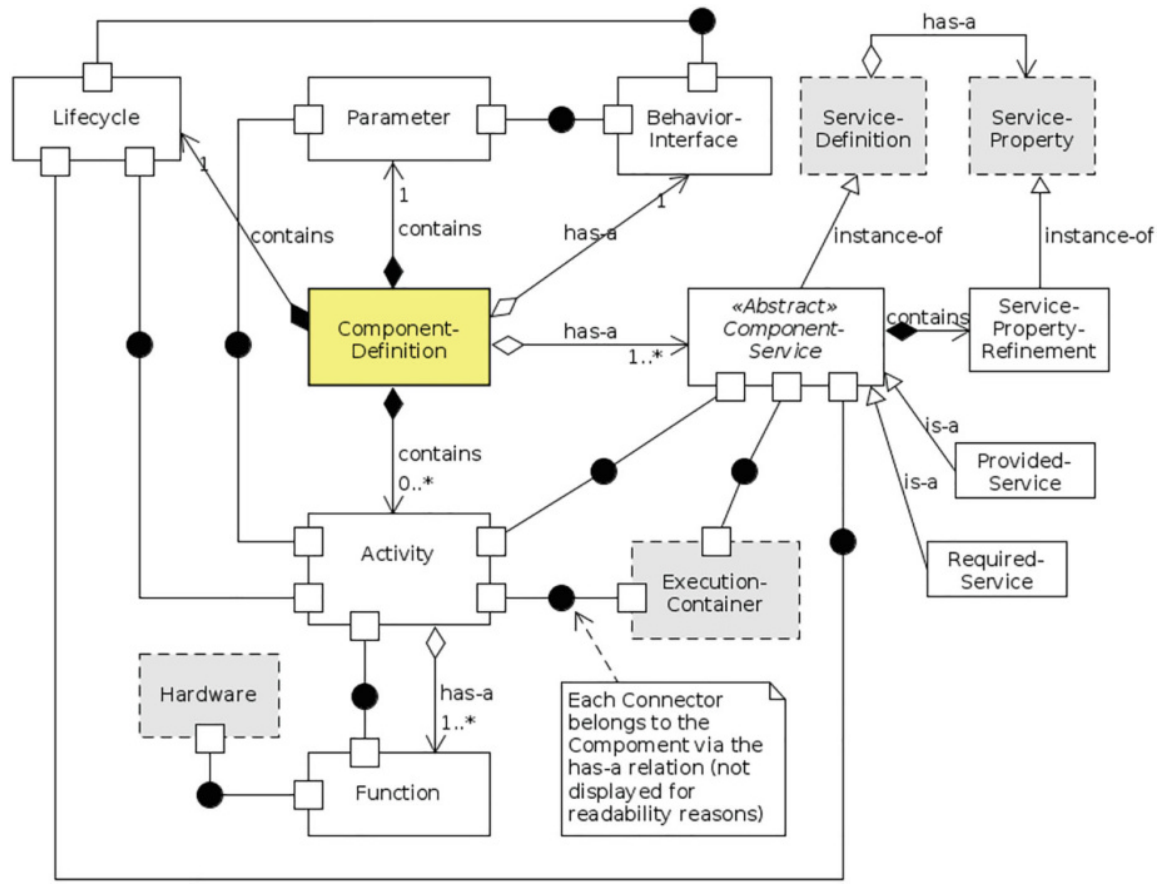

Fig. 3.12 The component definition meta-model shows the structure of a software component. A dashed gray box is a reference to another definition meta-model. The behavior of a block is defined by the behavior of its ports. A port is a block as well. A block refers to a behavior specification. A behavior specification can be in different forms (textual description, reference implementation, formalized model). 

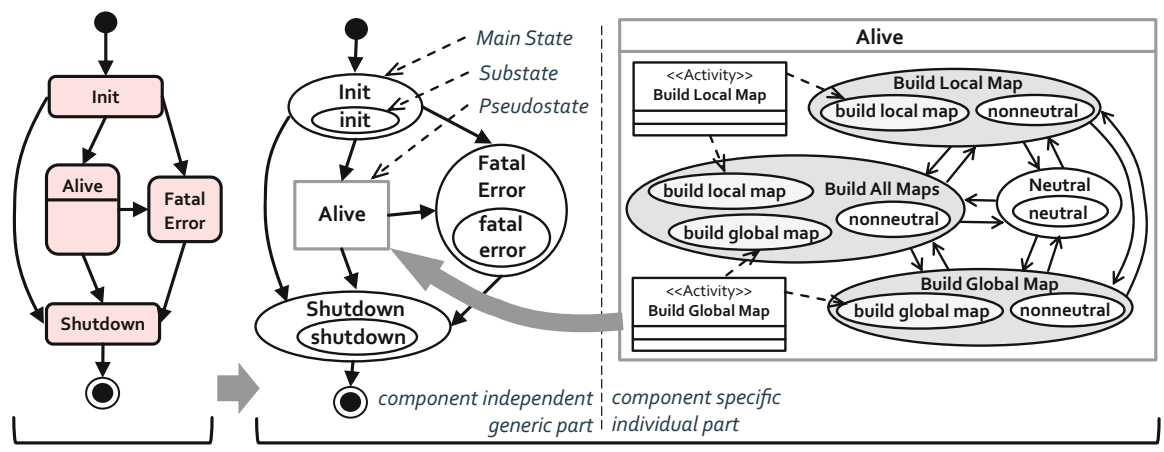

Fig. 3.13 The lifecycle of a software component with main states (visible outside), substates (inside coordination) and the pseudostate Alive (customizable).

with the Services of a component as their availability and their responses depend on the state of the component. It interacts with the Behavior Interface as this is the access from outside to the state of a component, its configuration and its monitoring (control flow interface).

The very concrete structure and behavior of the lifecycle of a software component are specified in detail in [26]. Figure 3.13 illustrates selected aspects. The lifecycle comes with the predefined main states Init, Shutdown, Fatal Error and Alive. At any point in time, a component can be in exactly one main state only. Main states come with substates. The main states Init, Shutdown and Fatal Error are created with exactly one substate each. As a convention, each name of a main state begins with a capital letter and each name of a substate begins with a small letter.

Activities inside a component refer to substates only and not to main states. An activity runs only when the required substates are active. From outside the component, only main states (and no substates) can be commanded. The reason for this approach is to decouple component internals from the outside view such that one can provide a standardized way of how to configure, control and monitor a component via its standard behavior interface. The approach becomes clear with the main state Alive, which is basically a pseudostate.

The main state Alive can be replaced by an individual state automaton that gets executed when the component is in the main state Alive. One can define an arbitrary set of substates for use by the activities within the component. The substates are clustered into user-defined main states. A substate can belong to any number of user main states. In this way, a user main state represents such a set of substates that can be consistently active at the same time and without conflicts. From the outside view, one needs to know neither the names of the individual substates nor the combinations of substates that can be consistently activated. One just needs to query the names of the user main states. One can set any of those user main states instead of the main state Alive, in any order. 
Of course, as the lowest layer of Tier 1 already defines a lifecycle automaton for a software component, one cannot just define another one and put it next to the already existing one. Nevertheless, there can be ongoing discussions within the expert group shaping the lowest layer of Tier 1 whether one wants to have different kinds of lifecycles that then coexist (which means to explicitly agree to go for fragmentation, perhaps, as an interim solution). In particular, the pseudostate Alive provides a hook for other individual state automata. Nevertheless, alternative proposals should also properly deal with the challenge of providing a standardized behavior interface, a consistent way to manage activities and communication according to states and state changes and others.

A different representation of a software component (that fully conforms to the component definition meta-model) is shown in Fig. 3.14. It shows different rolespecific views in a single figure. In the case of being a user of a software component, we see its outer view, which just comes as a block with ports as indicated by the solid surrounding line. These ports form the stable interface to other components and they are either a provided service or a required service. In the case of being a component developer, we see the stable interfaces of the inner part of the component

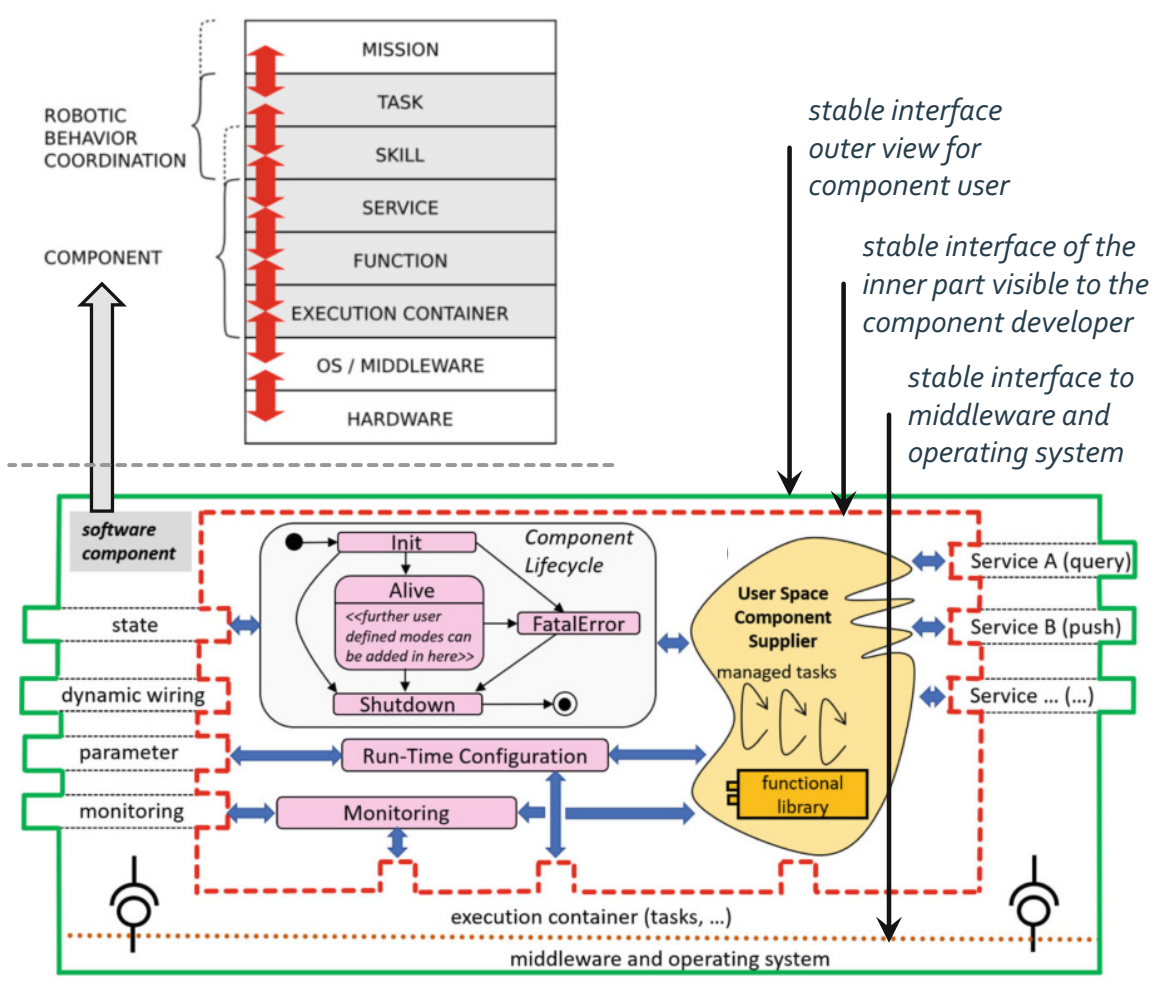

Fig. 3.14 Software component with communication (service-oriented ports), configuration (resources, parameters), coordination (modes, lifecycle) and computation (managed tasks). 
(dashed line). Finally, the dotted line is the stable interface to the middleware and the operating system. This is an internal interface hidden from the user. It links the execution container to the middlewares and operating systems and ensures that the execution container is agnostic to middlewares and operating systems.

If we open the software component block, we see that its internals are again blocks with ports. In particular, a component service (which is a port of the software component block) itself becomes a block (the complete component service) with ports. Its first port forms the external dock of a component service (visible from outside the component as component service port-solid line). The second port forms the internal dock of a component service (visible inside the software component only and only by the component developer-dashed line). Other ports form the internal interface to the component lifecycle and to the execution container (that links to the OS/middleware).

As the basic structure and behavior of a software component is defined at Tier 1, these are prescribed for Tier 2 and for Tier 3. However, the software component model still provides enough freedom when it comes to filling in, for example, domain-specific resource management mechanisms inside the dashed block. Of course, when domain-specific refinements become of general use, they can be moved upward to Tier 1.

Basically, the model-driven tools are the means to come up with proper presentations of role-specific views. For example, the model-driven tools then ensure that we select ports of a component only from a palette that represents what has been agreed upon in this domain and that we cannot use others not specified for the selected domain. The guidance and consistency of what is allowed in which step by which role as it is given by the model-driven tools stems from the meta-models and the reference implementations linked to the elements.

\subsection{Communication Patterns and Services}

The link between the component internal ports (dashed interface in Fig. 3.14) and the component external ports (solid interface) is realized via communication patterns. Basically, they cover a request/response as well as a publish/subscribe interaction. Table 3.1 gives an overview of all the communication patterns used by a software component for communication and for coordination. The patterns for coordination are based on the patterns for communication. The meta-model for the small set of generic communication patterns is shown in Fig. 3.15.

A communication pattern always consists of two complementary parts. The outer docks of complementary communication patterns can be connected with each other in case the used communication objects are the same. Again, it is important to note that all patterns come with well-defined structures (i.e. the APIs for their docks visible inside a component and also the definition of what is a fit between external docks) and well-defined behavior (i.e. the fully specified behavior of their externally visible dock and of the API of their internally visible dock). That is given by both 
Table 3.1 The communication patterns and the configuration \& coordination patterns of the software component model (see [21, metamodels:commpattern])

\begin{tabular}{l|l|l|l}
\hline Pattern name & Interaction & Description & Definition \\
\hline Send & Client/server & One-way communication & {$[27, \mathrm{pp} .85-88]$} \\
\hline Query & Client/server & Two-way request/response & {$[27, \mathrm{pp} .88-96]$} \\
\hline Push & Pub/sub & 1:n distribution & {$[27, \mathrm{pp} .96-99]$} \\
\hline Event & Pub/sub & 1 :n asynchronous condition notification & {$[27, \mathrm{pp} .103-112]$} \\
\hline Parameter & Master/slave & Runtime configuration of components & {$[17,28]$} \\
\hline State & Master/slave & Lifecycle management, modes & {$[26,29]$} \\
\hline Dynamic wiring & Master/slave & Runtime (re-)wiring of connections & {$[27$, p. 112] } \\
\hline Monitoring & Master/slave & Runtime monitoring and introspection & {$[28,30]$} \\
\hline
\end{tabular}

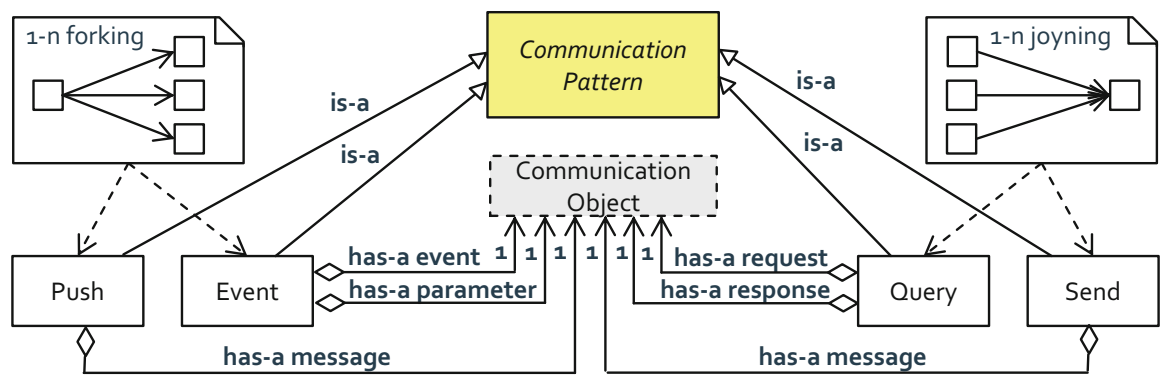

Fig. 3.15 The meta-model for the communication patterns (Communication Pattern Meta Model).

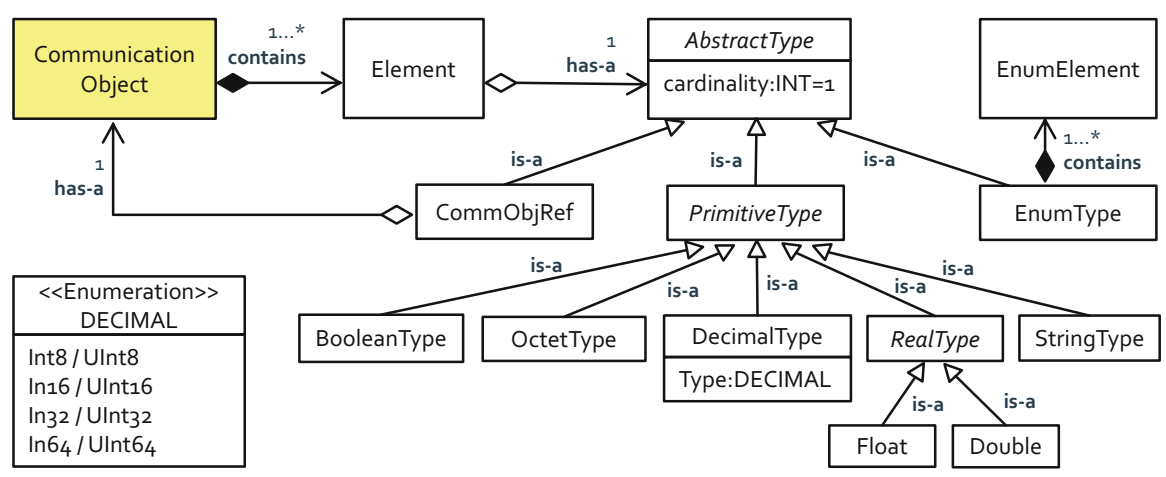

Fig. 3.16 The meta-model for a communication object (Communication Object Meta Model).

(1) descriptions in the form of documents [27] (that contain behavior specifications in the form of automata, sequencing diagrams, etc.) and in the form of API specs [31] and (2) reference implementations producing that very semantics (e.g. one such fully conformant implementation is given by SMARTSOFT/ACE [32]). 


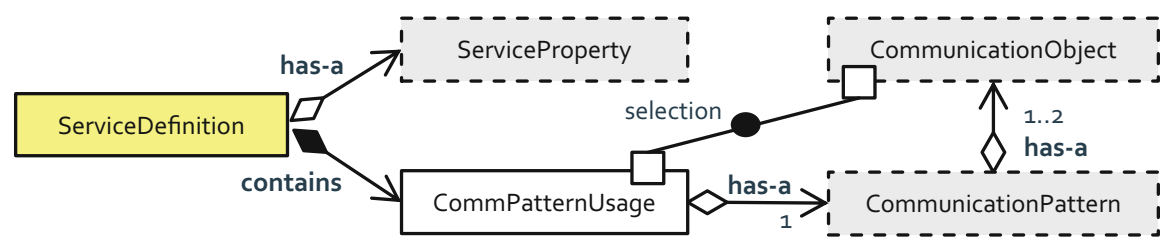

Fig. 3.17 The meta-model for defining services (Service Definition Meta-Model).

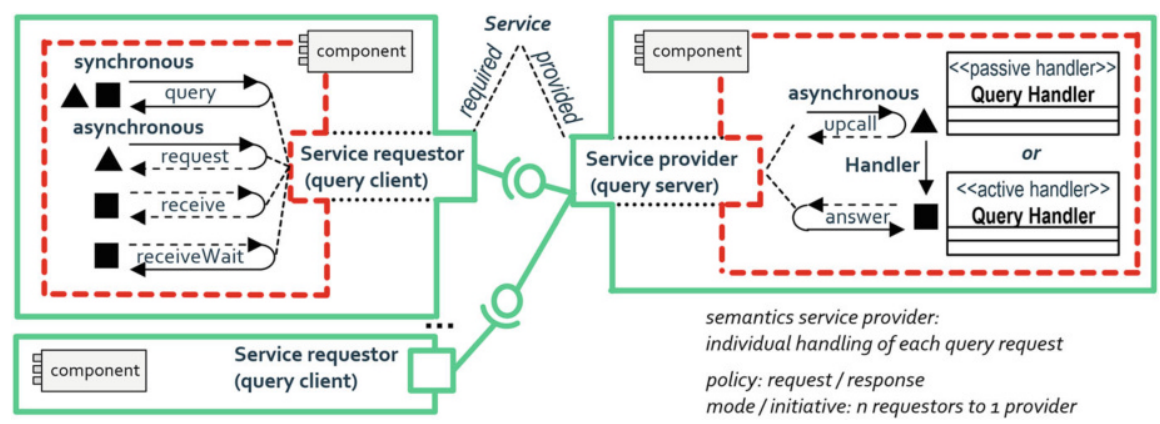

Fig. 3.18 A service based on the query communication pattern with the internal and external docks.

Figure 3.16 shows the meta-model for communication objects, and Fig. 3.17 shows the meta-model of a service. A communication object is a digital data representation that can be transferred by-value via communication patterns. A service is the combination of a particular communication pattern with selected communication objects. Thus, a service shows exactly the structure and behavior as defined by the used communication pattern, although it is customized via the used communication objects. A service is split up into a provided service and a required service according to the complementarity of the used communication pattern. Each port of a software component then is either such a provided service or a required one.

This is illustrated in a summary in Fig. 3.18. The shown service is based on the query communication pattern. The provided part of the service is based on the query server part of the query communication pattern. Accordingly, the required part of the service is based on the query client part of the query communication pattern. It shows also the interfaces of the internal docks that are visible to the component developer. The client-side access policy (synchronous, asynchronous) is completely decoupled from the server-side processing policy (asynchronous via handlers). 


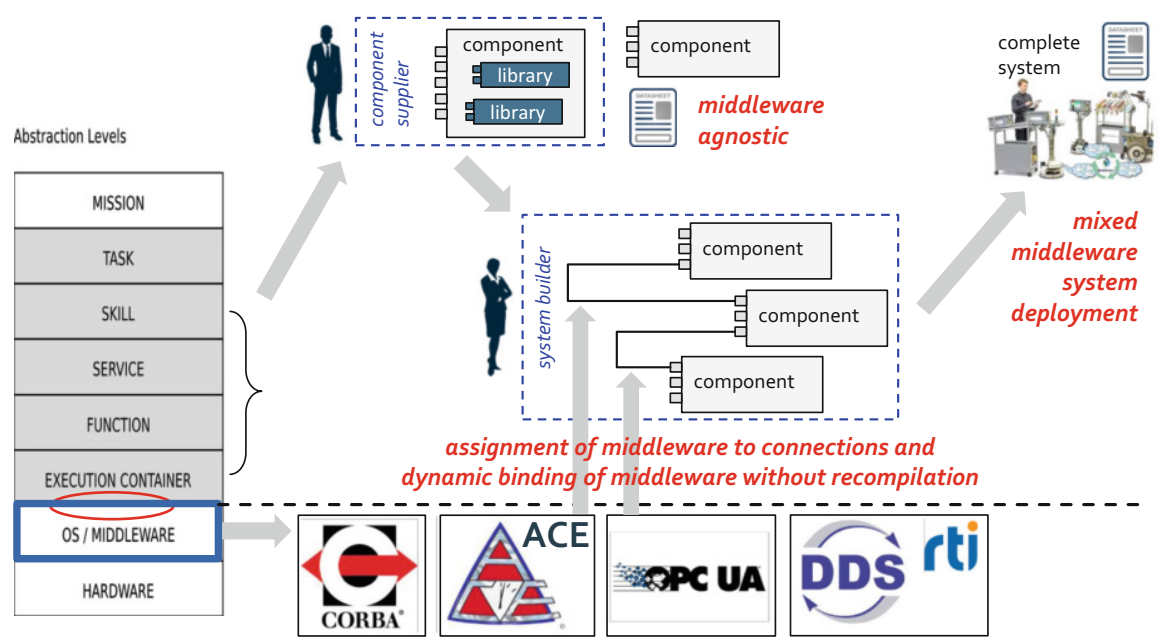

Fig. 3.19 The interface between the abstraction layer execution container and the abstraction layer middleware/operating system allows for middleware-agnostic software components.

\subsection{Middleware-Agnostic Software Components}

The structure and behavior of the communication patterns and of the communication objects, and thus of services as well, are completely middleware-agnostic. Their semantics is defined fully independently from any particular implementation technology. It is at the interface between the layer of the execution container and the layer of the middleware/operating system (Fig. 3.19) where the given and specified semantics of the communication patterns must be reproduced exactly as specified via the means of the selected underlying middleware technology. It is also at that interface where the elements of the communication object meta-model get mapped onto marshaling mechanisms of a middleware. The mapping between the execution container and a particular middleware/operating system is provided by experts on system-level software. Experts for model-driven tools make use of such mappings and reference to them from within their tools to generate artifacts for the according middleware/operating system.

By means of a common meta-model with a clearly specified semantics, one can replace a middleware by another one without impact on the overall behavior of the system. One can even mix different middlewares within a single system and one can decide on a middleware per connection. With dynamic linking, one can do this even at deployment time and without recompiling any of the components. A common meta-model also allows for the automatic transformation between different middleware-specific representations of the same communication object. Section 4.4 shows this via the SmartMDSD toolchain. 


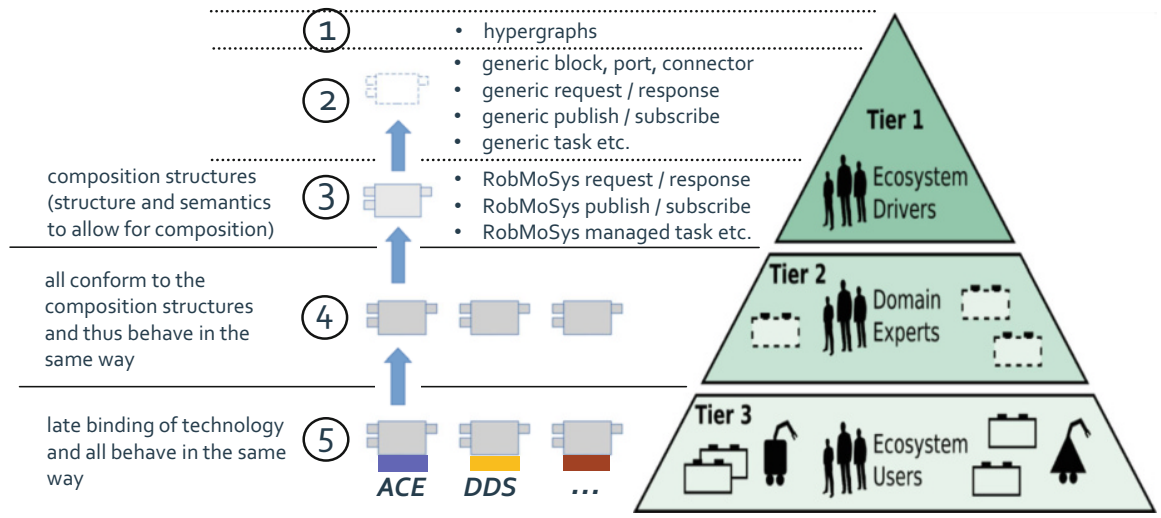

Fig. 3.20 Early binding of semantics and late binding of technology (not early binding of technology with lock-in into their semantics).

\subsection{Early Binding of Semantics, Late Binding of Technology}

Middleware-agnostic software components are a good example of the importance of the principle of early binding of semantics but late binding of technology. Only adhering to structure and ignoring the behavior destroys composition and thus results in fragmentation. The same happens when one just ignores the abstraction layers. Although that insight is a commonplace, there often is a tremendous lack in discipline when it comes to adhering to it.

Figure 3.20 illustrates this along the hierarchy of the tiers of an ecosystem. The composition structures (see (3), bottom level of Tier 1) are fully technology-agnostic (also agnostic to technologies of workbenches for model-driven tools). They ensure composition, composability and compositionality. These properties are available earliest at (3), which sets the frame for Tier 2 (see (4)). Whatever domain-specific structures are introduced at (4), these are consistent with (3) and they do not destroy composition. The same holds true for all the assets (see (5)) as these conform to (4). The behavior of a structure introduced at (3) frames the refinements at (4) and (5). Late binding of an implementational technology at (5) (such as a particular middleware) does not change the behavior given by (4) (and given by (3) as that binding is eligible only when it produces a behavior exactly in line with (4) (which is then also in line with (3). In this way, all the assets at (5) are not affected in their behavior when we move to another underlying implementational technology and there are nearly no migration costs.

In contrast to that is the approach of directly working with a particular implementational technology and let that subsequently drive structures and behaviors. Any change of the implementational technology would affect the behavior as well. This easily results in huge migration costs with any new technology and obviously is in conflict with the aim of composability. Therefore, it is also a misunderstanding that arbitrary solutions, which are available so far in source code only, can become 
part of the ecosystem by just modeling them. The models in Tier 1 are much more as they ensure composition, composability and compositionality.

Thus, the following often-seen proposal for extending the ecosystem structures does not help to attain the objectives of a business ecosystem. A typical but inappropriate approach is that an expert for a particular robotics domain or a particular software framework just picks out an arbitrary block from the ecosystem structures and ignores its behavior. A typical example is to select the generic request/response block from (2) and to refine that into the query of a particular framework and provide its behavior by the implementation from that very framework. In consequence, it becomes an alternative to what is already there at (3). However, (3) defines a query that ensures composition, composability and compositionality. Either the new proposal comes with those properties as well (then it would be very astonishing if it is different from what is already there), or it does not come with those properties (then it should not be placed at (3) as then Tier 2 and Tier 3 cannot rely on composition anymore). It can also not be placed at (4) as this would not be consistent with (3). Just placing it at (4), skipping (3) and extending (2) is also not an option as this again circumvents the composition structures and thereby jeopardizes the ecosystem foundations.

Of course, one can still propose to put another query at (3) that does not come with all the properties required for composition. However, such a query then does not help the ecosystem objectives. This is the same for all the other models such as the lifecycle of a software component, its behavior interface and many others. Again, model-driven tools can enforce compliance where necessary and it can circumvent relying on discipline only.

\subsection{Horizontal Versus Vertical Composition}

While vertical composition addresses the composition of parts located at different layers of abstraction, horizontal composition focuses on the composition of parts located at the same layer of abstraction. The concepts are illustrated in Fig. 3.21.

The difference becomes obvious in the context of control hierarchies. In a control hierarchy, resources assigned to a particular layer can be managed there. As long as one operates within the given boundaries (constraints on the assigned resources), there is no conflict with others. In particular, we can assign shares of our resources to entities that are under our control (vertical relationship). These then can again manage those resources within the given constraints and according to the given policy. It gets more complex in case we need access to resources outside our responsibility. In such a case, we need to come up with a contract that secures that horizontal relationship. 


\subsection{The Data Sheet}

Figure 3.22 shows more details of a digital data sheet using an the example of a software component. The very same principles apply for all the assets in an ecosystem.

A digital data sheet comes in digital form and it contains different parts: at least a technical part that is generated from the technical models of the asset and a descriptive part that comprises manual annotations done by humans. The manual annotations in the descriptive part can range from free-form text fields to ontologybased labels. A data sheet is a model and thus its form, content and meaning are defined at Tier 1 (general parts) and Tier 2 (domain-specific parts).

The technical part is more like a technical product data sheet, operating manual, etc. It is technically binding and states guaranteed properties that can be claimed by customers. It supports aspects such as what-if analysis, prediction, composition, configuration and runtime adaptation. As can be seen in the lower right, the technical part may also contain an abstract representation of selected internals such as data flows and transfer functions needed for analysis, checking, predicting and configuration of system-level properties.

The descriptive part is more like a product description for presentation in a web store or in an advertisement. Its focus is on finding and preselecting assets from a marketplace. Thus, the descriptive part is more market-oriented, oriented to gain attention and awareness, and thus is more user-oriented. The statements in the product description are mapped to and grounded by the technical part of the data sheet.

The concept of a digital data sheet is fully consistent with the concept of an industry 4.0 asset administration shell (AAS). As shown in the upper right-hand side of Fig. 3.22, the digital data sheet can become just a submodel in the AAS and thus part of the AAS container.
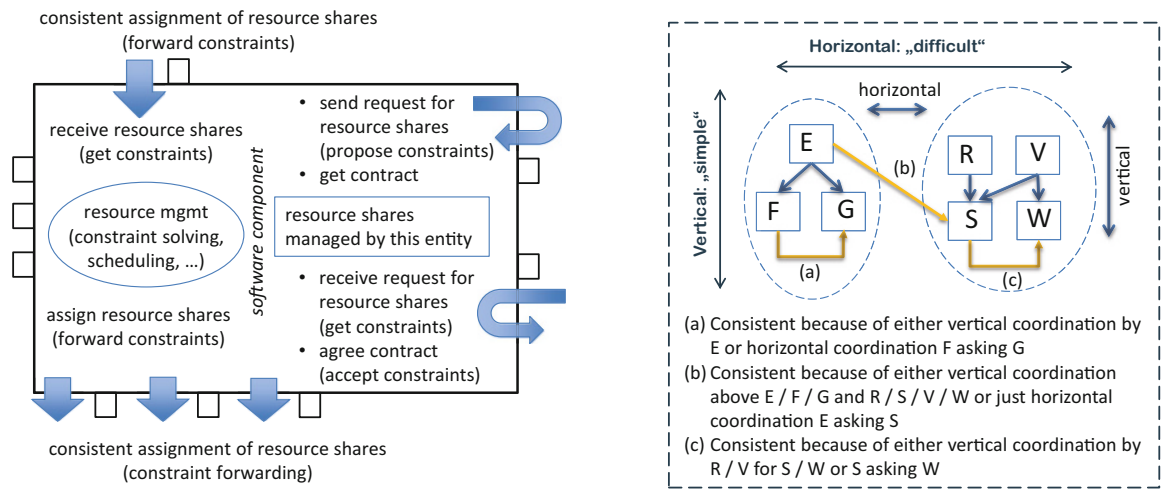

Fig. 3.21 Horizontal versus vertical composition: separation of control flow and data flow and responsibilities for resources. 


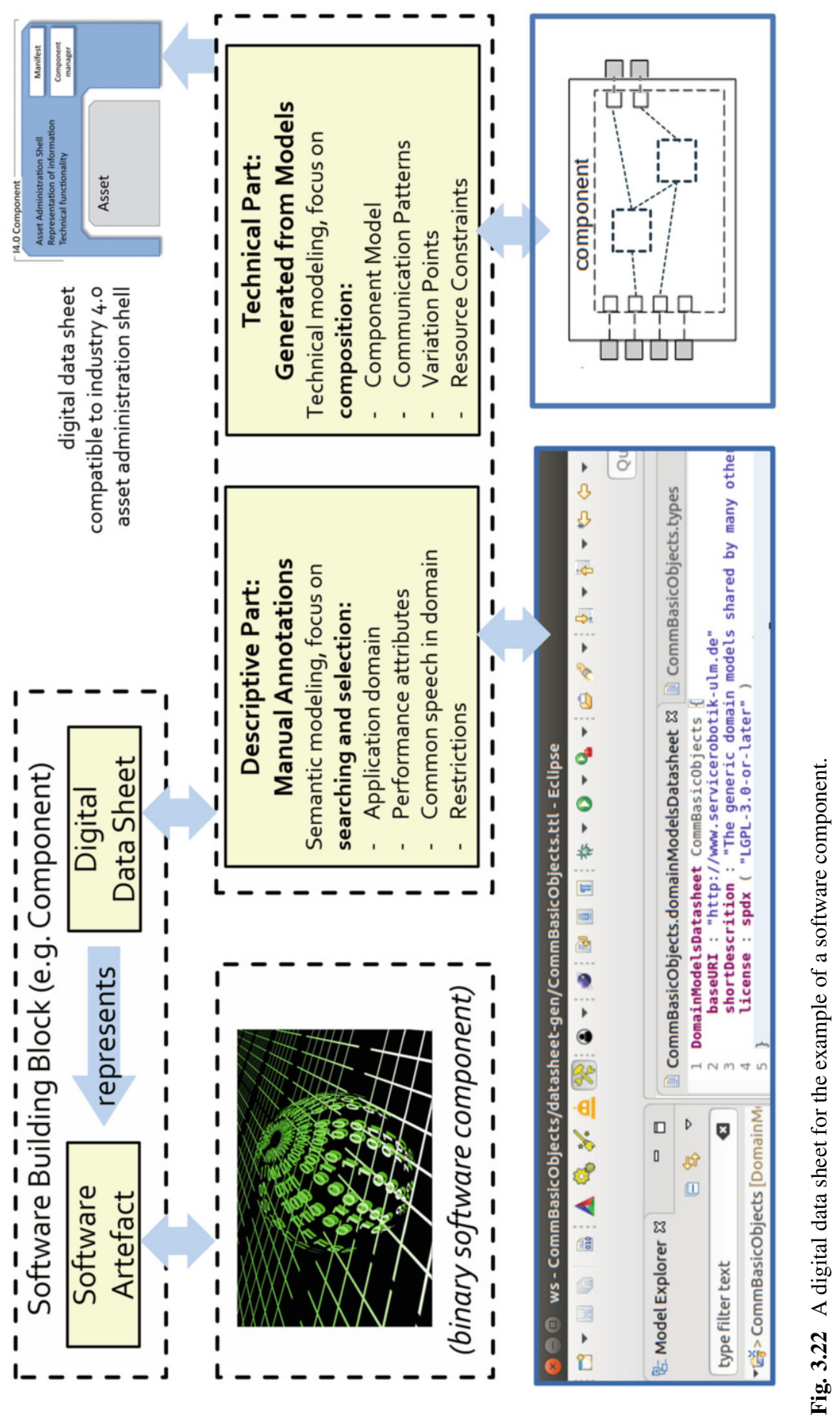




\subsection{Dependency Graphs and Constraints on Services}

Dependency graphs can model system-level requirements that span across different components. Examples are properties along data flows, such as quality and ageing of data, but also consistency aspects, triggering along computational chains and arrival time analysis [33]. A dependency graph can also be used to express requirements on data privacy, for example, "there must not be any link between a service providing a raw camera image and a service connecting to the network outside the robot". A dependency graph can also express that, for example, the blur of a camera image depends on the speed of the robot. In order to keep the blur below a given threshold, one can derive the related maximum allowed velocity. A dependency graph can guide the system builder in selecting and configuring components according to the input from a system architect, a safety engineer and others. Thereto, the foreseen variation points, explicated in the data sheet, are exploited.

Dependency graphs can also be extracted from a virtual system composition (based on data sheets only; see Fig. 3.7, Sect. 1.5). Before building the real system, one can already check whether the dependency graphs in the virtual system comply with the constraints of the dependency graphs representing the requirements. Tools can be used to try different configurations and to check for matches. In that way, dependency graphs also help to implement traceability from requirements to fulfillment by configurations of variation points. Dependency graphs also serve at runtime as sanity checks (before finally implementing a runtime decision) and for monitoring integrity.

From a technical point of view, the meta-model of a dependency graph is again based on the entity-relation model provided in layer 2 of Tier 1, which refines into different variants at layer 3 of Tier 1. The dependency graph shown in Fig. 3.23 is a simple one that expresses dependencies between services (entity service, relation uses). It gets checked by a mapping between the dependency graph and the graph resulting from the data sheet composition.

Figure 3.24 shows two more examples of a dependency graph. The example on the left shows error propagation through a data flow across components. Transfer functions of a component specify what additional uncertainty comes on top. For example, the system builder can check whether the selected component is good enough to match the requirement at the end of the processing chain.

The dependency graph on the right is based on name/value pairs. The left component might be a service providing a camera image. That data is labelled as critical with respect to data privacy. The right component might provide a map based on the camera image (the transfer function for this port makes critical input to uncritical output) and the camera image enriched with pose information (critical input results in critical output at this port). Interval arithmetic and constraint solving are further mechanisms for dependency graphs. Trigger chains are another example that is illustrated in more detail in Sect. 4.6. 

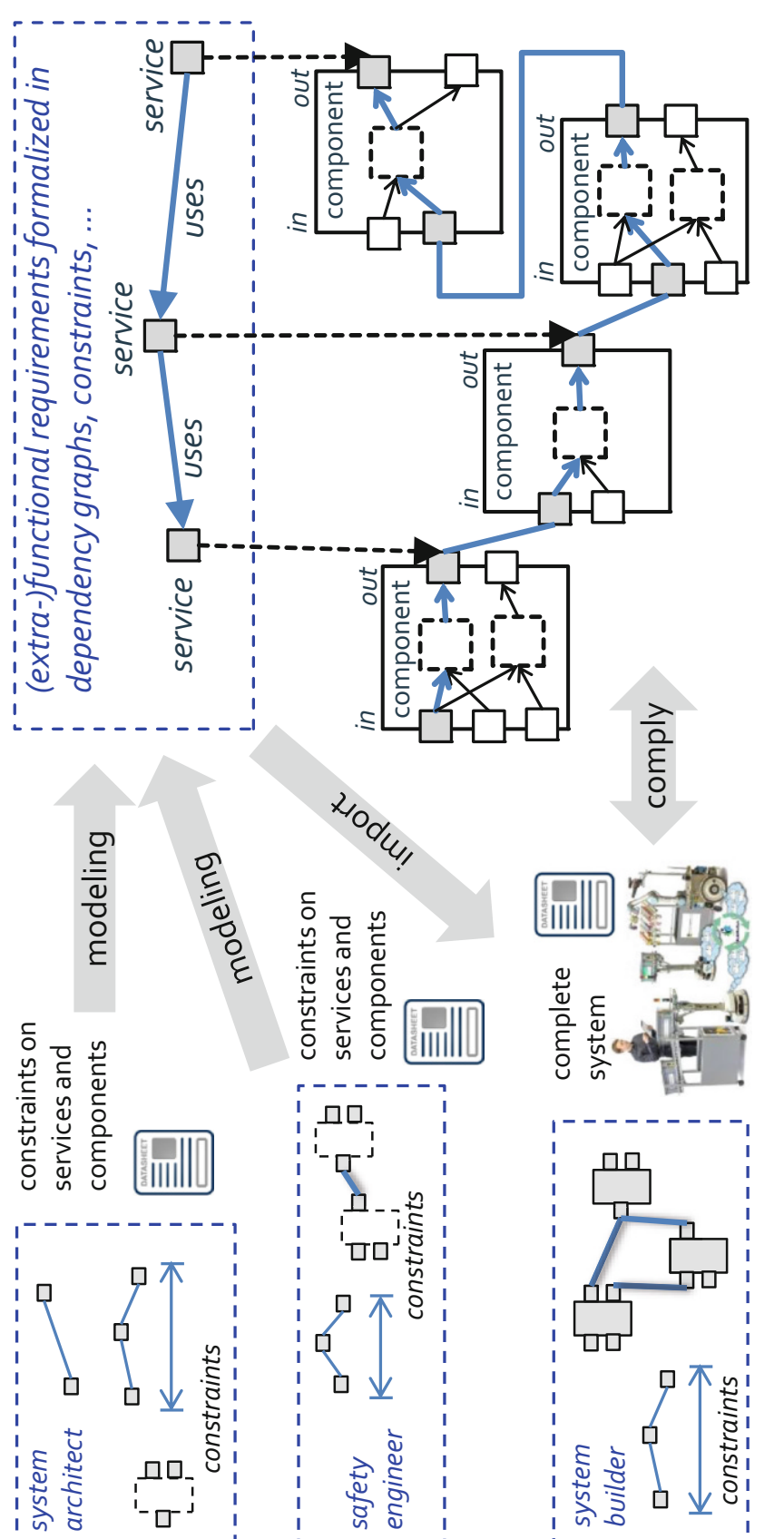

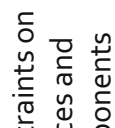

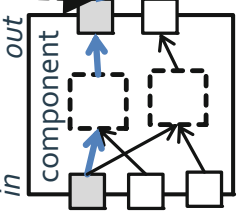

흘

을

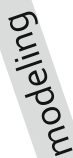

气 $\frac{\cup}{2}$
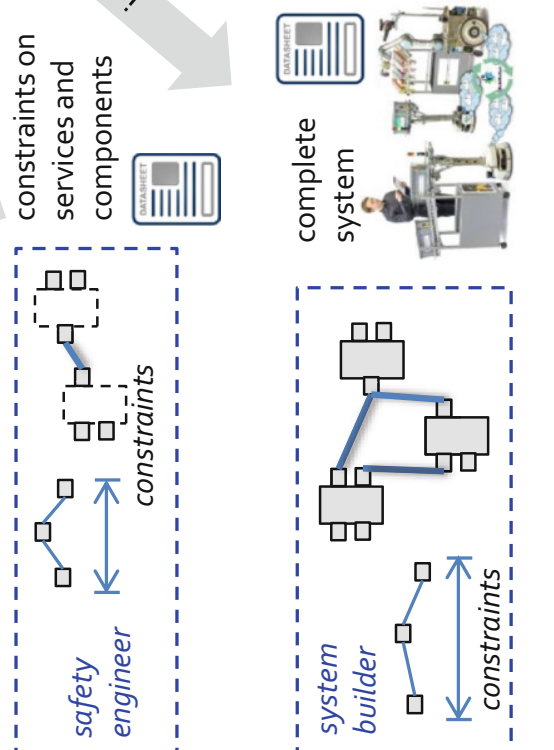$$
\text { 年 }
$$

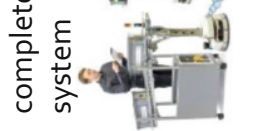

है
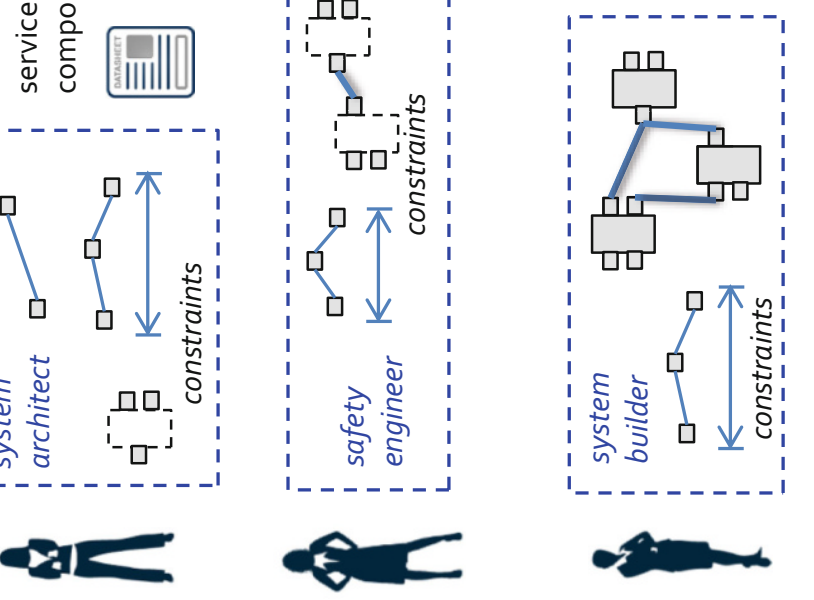

这 

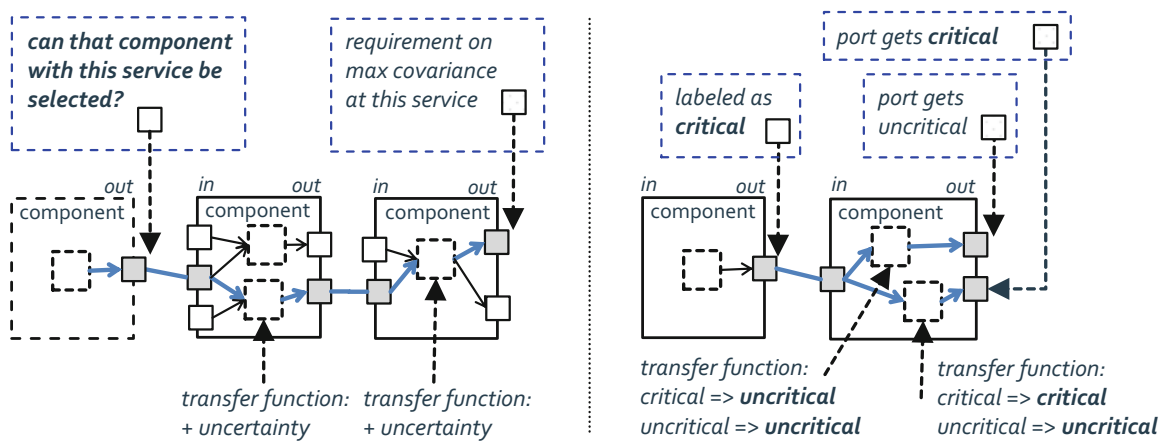

Fig. 3.24 Further examples of dependency graphs. Left: checking component selection (error propagation). Right: checking criticality of information (name/value pairs).

\subsection{Tasks, Skills and the Behavior Interface}

Robotics behavior blocks are separated into different levels of abstraction in order to allow for composition and for separation of roles. A skill provides access to functionalities realized by components. It is the bridge between generic descriptions of capabilities (independent of a particular implementation) and behavior interfaces (configuration-resources, parameters, wiring; coordination-modes, lifecycle, events) of a (set of) component(s) to achieve that capability (specific to the used components). A skill lifts the level of abstraction from a component-specific level to a generic level. Thus, different implementations for the same capabilities become replaceable as they are accessed in a uniform way.

Tasks describe via which steps (what, the ordering of steps) and in which manner (how, the kind of execution) to accomplish a particular job. This is done in an abstract manner independent from a particular robot as tasks refer to skills for their grounding (see also Chap. 12 on mission specification).

Tasks and skills are domain-specific as they refer to domain-specific vocabularies. Nonetheless, software components can be used in skill sets of different domains. Of course, one can also define a domain that holds generic tasks and skills that can then be used from within different domains.

We illustrate that for the domain of intralogistics. A task for order fulfillment arranges several other tasks such as move to a shelf, locate and pick an item, follow the worker and deliver the item at a packing station. Such tasks refer to skills for their execution, for example, to skills such as navigate-to, recognize-object and grasp-object. A particular task can be executed by a particular robot when the robot possesses matching skills for all the required skills. For example, a task might refine into a skill for grasping an object. From the execution context and its bindings, one knows that the object has a maximum weight of $1 \mathrm{~kg}$. The robot can select any of its grasping skills that can handle at least $1 \mathrm{~kg}$. 


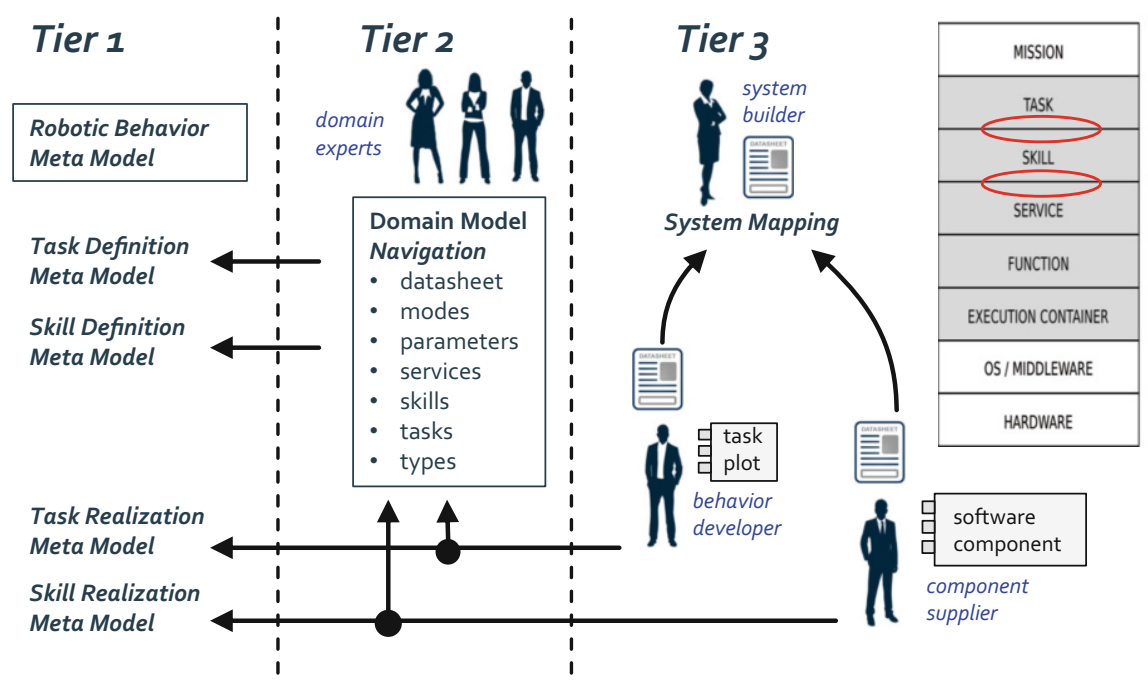

Fig. 3.25 The general context of meta-models for tasks and skills and their link to the behavior interface of software components.

Figure 3.25 illustrates the general context and selected roles, namely, domain experts, component suppliers, system builders and behavior developers. It also comprises different meta-models from Tier 1 and their use at Tier 2 and Tier 3. Skill definitions are being done by domain experts that belong to Tier 2 of an ecosystem. Those skill definitions are prescriptive for component developers that operate at Tier 3 of an ecosystem. A component developer adheres to the skill realization metamodel in making accessible the capabilities provided by its component (including references to other components needed for that capability) and thus does this in full conformance with the Tier 2 domain-specific specifications. Finally, the behavior developer is able to model task plots and ground them in domain-specific skill definitions. The skill definitions are the interface to software components providing the required functionalities and getting them executed. The components selected and composed by the system builder to form the robotic system all come with their individual skills. These skills get listed in the data sheet of the robotic system. Thus, the set of skills available on a concrete robotic system is part of and available via its accompanying data sheet.

Selected meta-models of Tier 1 for the behavior interface are shown in Fig. 3.26. The overall link between tasks, skills and services of a software component is organized by the robotic behavior meta-model. The task definition meta-model and the skill definition meta-model are the entry points to provide the means for the modeling views of the domain experts at Tier 2 . They describe which elements form sound descriptions of a task and of a skill. They specify that input values and output values as well as results are described by attribute-value pairs. A result consists 

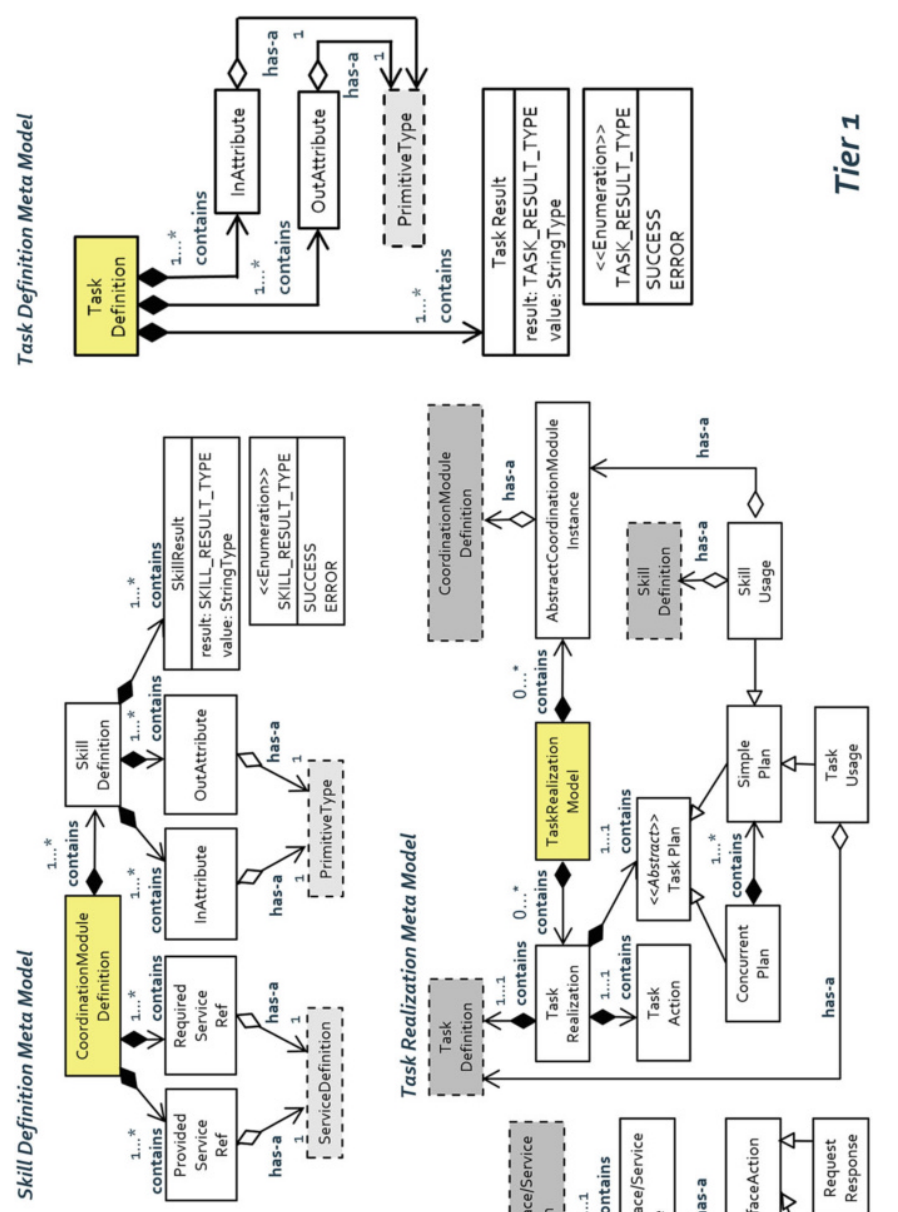

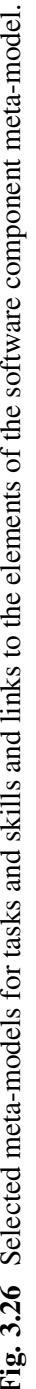


of a list of string values, each labelled with either success or error. Task and skill definitions are grouped into sets to model their semantic cohesion.

Of course, the domain-specific definitions of attribute names and type assignments for input/output values as well as the domain-specific set of possible string values for results are defined by domain experts (Tier 2). It is at Tier 2 where all the tasks and skills that are possible within a particular domain are defined. That is done by modeling them in a way that is conforming to the definition meta-models for tasks and for skills. Based on the generic concepts at Tier 1, domain experts model for their domain the concrete forms of data sheets, tasks and skills. They model the concrete sets of communication objects and services, and they also define concretizations of configurations (configurable parameters and configurable main states).

The domain-specific task and skill definitions at Tier 2 ensure that different components with all their specifics present their capabilities in the same way. In consequence, skills belonging to the same domain represent the same capability when they use the same name and their input/output arguments as well as their result values follow the same domain-specific vocabulary and meaning.

The task realization meta-model and the skill realization meta-model put all those domain-specific models together. They form the foundation for presenting aspects of robot behavior in role-specific views at Tier 3. For example, software components comprise a behavior interface. Its generic structure and semantics is part of the component definition meta-model (Fig. 3.12, Sect. 3.1). It is used to configure control flows, even dynamically at runtime.

The component supplier needs to adhere to the domain-specific ports for software components (communication objects, communication patterns, services, parameterization, main states). The component supplier needs to model skills of its (set of) component(s) along the domain-specific agreements.

Task plots are modelled by the behavior developer. They refer to skill definitions, not to software components. The link between tasks/skills and skills/component instances on the robot is set up by the system builder.

More insights into role-specific views are given in Sect. 4.7. The differentiation between the task/skill definition meta-models and the task/skill realization metamodels is in the same vein as for communication objects and for services, where also the generic means are provided at Tier 1 and the domain-specific means are at Tier 2.

\section{Links Between Composition Structures, Roles and Tools}

This section gives further insights into some links between definition meta-models, realization meta-models and roles using views of them. This also gives insights into how the independent activities of different roles can result in consistent assets due to the overall guidance of the meta-models in the different tiers. Of course, the metamodels and role-specific views are independent from their implementation within 
a particular model-driven tool. However, examples are illustrated by screenshots from the open-source eclipse-based SmartMDSD toolchain [34], which implements the composition structures via Ecore. This is much more explanatory than going into details of their lengthy Ecore-mappings.

\subsection{The Role of the Domain Expert (Tier 2)}

Figure 3.27 show selected views for the Tier 2 domain experts. Types define communication objects, services define the combination of communication patterns and communication objects, modes define the agreed and configurable main states for software components, and coordination services define the coordination and configuration interface. Middleware-agnostic communication patterns require middleware-agnostic communication objects. The middleware-agnostic modeling of data structures is also used in a broader context and is then referred to as digital data modeling. In the context of communication objects, the digital data models are mapped in a semantics-preserving way onto the middleware marshaling mechanisms. Skill definitions define the task-level interface of skills and the data sheet refines the non-technical part of a data sheet (e.g. that license information is to be provided in that domain).

\subsection{The View of the Component Developer (Tier 3)}

Figure 3.28 shows a software component. The component developer selects from the domain-specific models at Tier 2 the ports (required and provided services) and the behavior interface (parameterization, configuration, main states of lifecycle, etc.) and models the skills of the component along a Tier 2 skill set. An example is shown in Fig. 3.29.

Skills always come with a component as a skill has a component-specific side (component behavior interface). A skill of a component can refer to skills of other components in order to allow for modeling skills that require a set of components. At the end, the set of models for a software component comprises the component model itself, the model for its parameters, its data sheet, its documentation and the skill realization.

\subsection{The View of the Behavior Developer (Tier 3)}

Figure 3.30 shows the SmartTCL version of the task realization model of a transportation task. SmartTCL is a task-coordination language for hierarchical task nets [35]. It comes with an interpreter, which at runtime dynamically expands tasks 


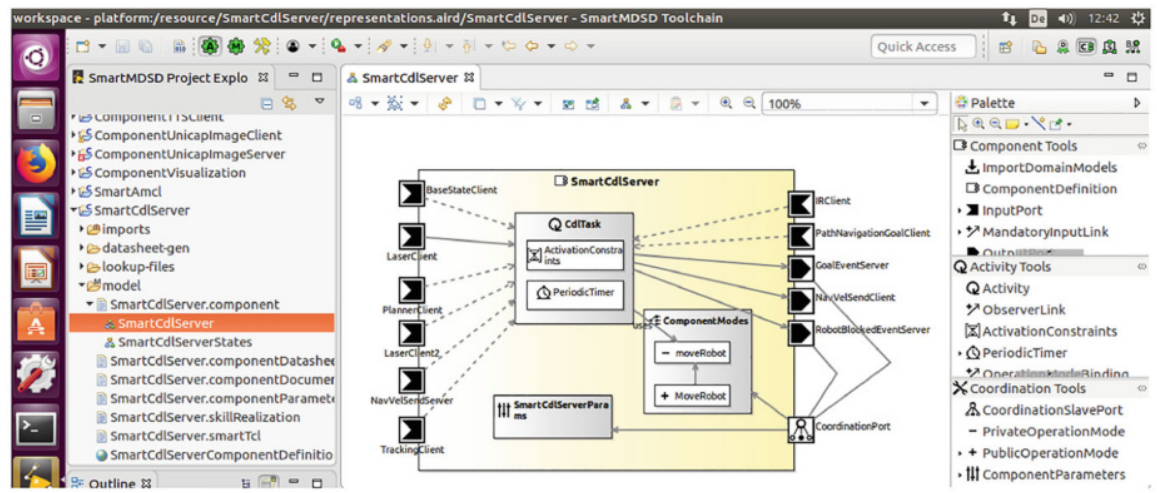

Fig. 3.28 The component developer view in the SmartMDSD toolchain (Tier 3).

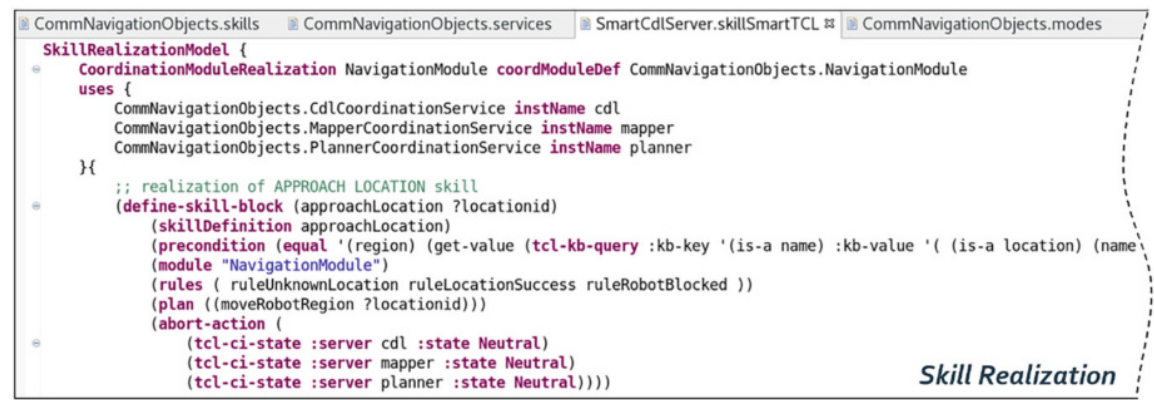

Fig. 3.29 Excerpt of the skill modeling for a software component (skill realization by the component developer in the SmartMDSD toolchain at Tier 3 using the SmartTCL representation).

into arrangements of other tasks/skills based on the current situation and context. Besides a hierarchical refinement (see the $t c l$-push-plan statement, in this example a refinement into skills), arrangements of tasks and skills can be sequential, parallel, one-of and others. The example nicely shows the binding of task variables via the knowledge base of the robot which, for example, contains the world model (linking names of rooms to regions in a particular map and a particular coordinate system) and also the data sheets of the used components. It is accessed as any other component via service ports (in our example, a service port for tell-and-ask expressions). The example also shows how a task model refers to skill realizations. Some more details on the relationship between design-time task and skill modeling and their runtime execution follow in Sect. 4.7. 


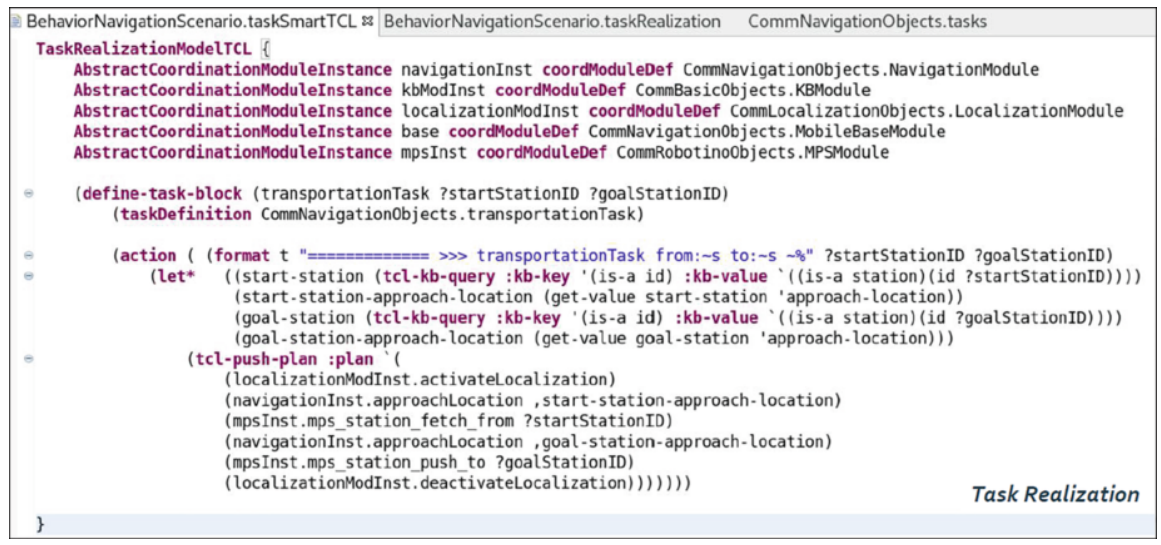

Fig. 3.30 Task model for a transportation task: realization by the behavior developer in the SmartMDSD toolchain at Tier 3 using SmartTCL.

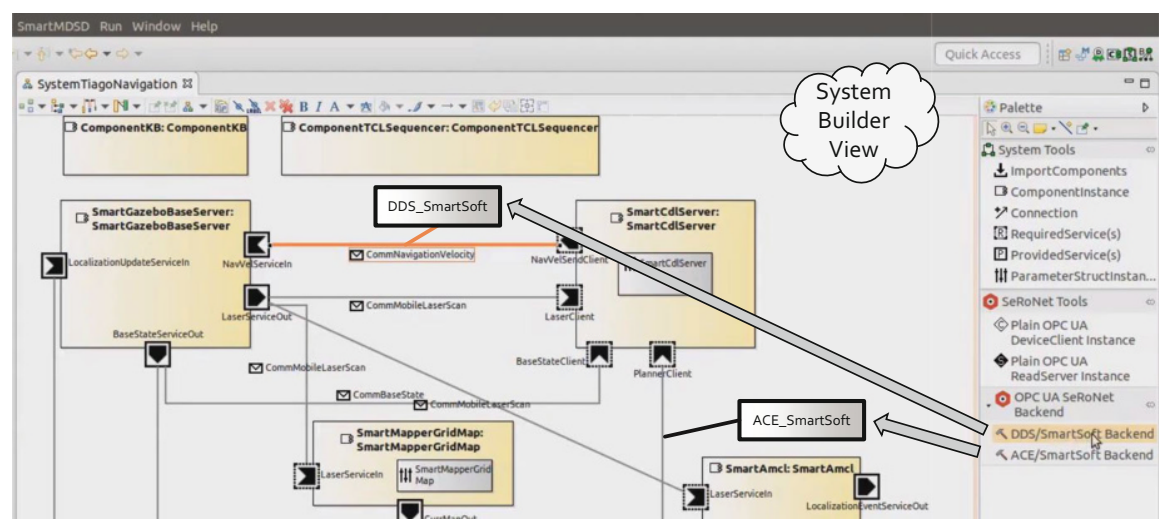

Fig. 3.31 Using different middlewares for different connections at the same time in a system.

\subsection{Middleware-Agnostic Components and Mixed-Middleware Systems}

The system builder view for selecting and composing different components is shown in Fig. 3.31. The system builder can select for each connection the middleware to be used. Different ports of a component can use different middlewares. Even different connections of the same port can use different middlewares. The components are not recompiled with a change of the underlying middleware as the links to the runtime executables of the middlewares are dynamically linked with the deployment process. 


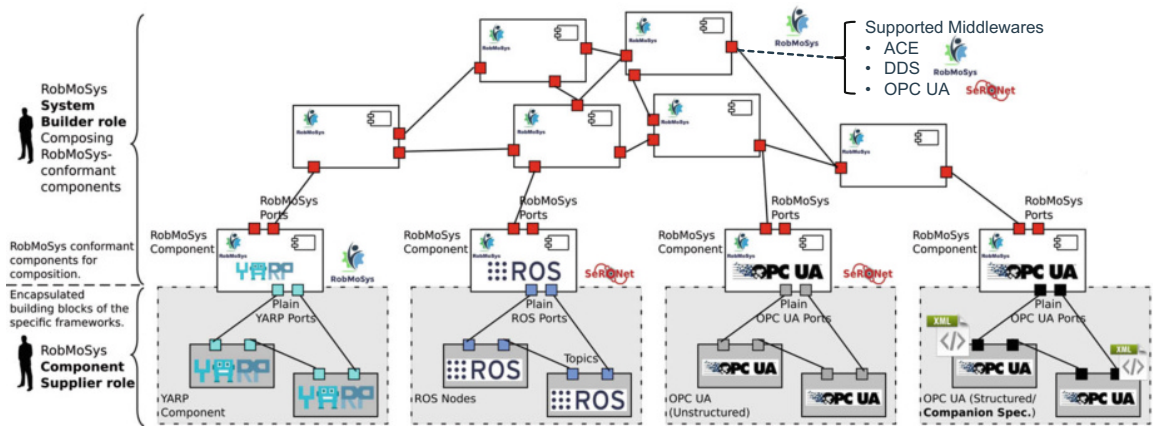

Fig. 3.32 Mixed-port-components serve as gateways to legacy systems and offer a migration path to the full-fledged composition structures.

\subsection{The Mixed-Port Component as Migration Path}

A smooth migration path from current systems to the full-fledged composition structures is very important to secure existing investments. Very often, we have to cope with a brownfield setting and thus need to be able to interact with our legacy parts. A mixed-port component shows two different sides of ports (Fig. 3.32). The first side consists of RobMoSys ports and the second side consists of ports of the legacy framework. A mixed-port component is like a gateway, which fully hides access to the legacy system. Thus, the resource management of the legacy system does not get in conflict with the RobMoSys composition structures (and their way of managing and configuring resource assignments). Relevant examples of mixed-port components are links to ROS systems and to industry 4.0 devices. The SmartMDSD toolchain comes with full support for ROS mixed-port components [36, start\#lesson_6interfacing_with_ros_subsystems] and for OPC UA mixed-port components [36, start\#lesson_5opc_unified_architecture_opc_ua].

It is important not to confuse mixed-port components with middleware-agnostic RobMoSys ports. For example, OPC UA can be used as middleware underneath RobMoSys ports. It is then fully hidden and just serves as a middleware to produce the semantics of the RobMoSys ports. In contrast, a mixed-port component for OPC UA offers at its second side native access to OPC UA devices (thereby, e.g. following OPC UA companion specifications).

\subsection{Deployment-Time Configuration of Trigger Chains}

In a robotics software business ecosystem, the development of a software component is first fully completed by a component developer before it is then offered $a s$ - $i s$ for use by others. In many approaches for robotics software components, the component developer ultimately decides how a particular task inside a component 
gets triggered (time-triggered such as periodically or event-triggered such as by incoming data) and then already invariably fixes that for the software component at the time of its implementation.

However, trigger chains (also called cause-effect chains) are a system-level property. They are a particular form of a dependency graph. Trigger chains occur in the context of data flows and typically map onto publish/subscribe communication. Trigger chains are part of the information architecture specified by the system architect.

The information architecture gets instantiated by a system builder by selecting software components that fit the information architecture and that match the dependency graphs. A software component will be extremely limited if it comes with prescribed trigger sources and thus only fits selectively. Thus, a software component must allow a system builder to configure the trigger sources for its activities. However, there should be no need at all to deliver the source code of a software component to the system builder and to have the software component recompiled. System builders neither want to go into internals of a software component nor are they expected to do so. Indeed, the data sheet of a software component shall contain trigger sources as variation points, which are then configurable without a need for recompilation.

For this use case, another abstraction layer is added inside a software component as refinement for an activity. This is just one example of how the internal structures of a software component can be refined or enriched with additional structures which form coexisting but consistent and dedicated offers for particular use cases. Further examples of such refining structures are scheduling mechanisms inside a software component or time-based approaches for functional composition.

The component developer now does not anymore write code that directly accesses the native API of ports providing incoming data. For example, the native API of a push client offers a getUpdate method (reading the latest available data without blocking) and a getUpdateWait method (blocking wait for the next update). Writing code using these access methods prescribes whether the access follows a register semantics (getUpdate) or a trigger semantics (getUpdateWait). Instead, the port is now accessed via a get method that forms a stable interface for programming independent from the subsequent trigger configuration. The get method is generated by the model-driven tools if the extension of trigger configurations is selected.

In a software component, the activity depends on a trigger. Thus, the additional abstraction layer is an extension of the activity. As shown in Fig. 3.33, the model of a software component comprises its ports and its activities, but also from which ports an activity reads its input and to which ports an activity forward its output. Model-driven tools generate from this model the component hull with its stable programming interfaces for use by the component developer.

An activity executes user code within its on execute method. The generated component hull includes all the mechanisms to configure an activity at any time to be either without trigger (calls the on execute method in a loop, user side selftrigger), periodic (calling the on execute method along the configured cycle rate and accessing all the ports with a register semantics) or input port (calling the on 


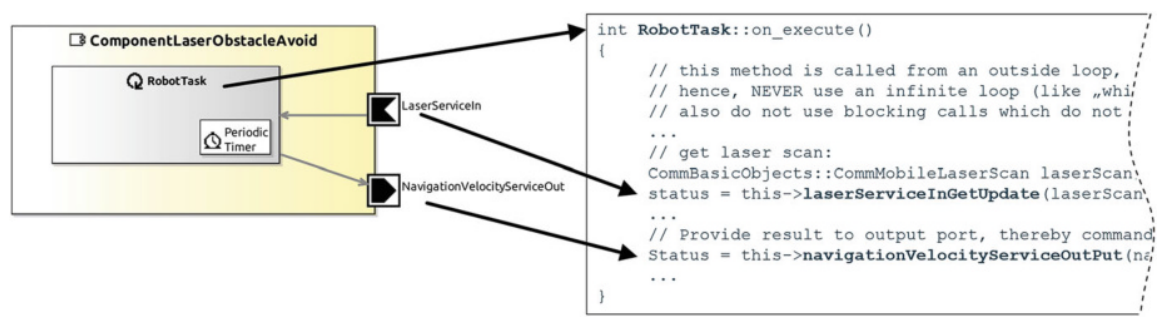

Fig. 3.33 The input port LaserServiceIn is of type 1-n forking (see Sect. 3.2) for which the access method laserServiceInGetUpdate is generated.

execute method only when new data arrives on the port selected as trigger port). This configuration then also sets the according mapping of the generated get method to either the getUpdate or the getUpdateWait method. The trigger for a task can be specified at any point in time: (1) the component developer can specify a default value, (2) the system builder can set it according to system requirements, and (3) the task-coordination mechanism can set it even at runtime.

Figure 3.34 shows an application for tracing and checking the consistency of data flows, trigger chains, response times and more. For example, one can ask whether the response time for reacting to obstacles along the cycle A-B-C-A is fast enough, whether it is fine that obstacle avoidance gets triggered with each new laser scan, or whether the mapper with its configured update pace is good along the loop A-B-DE-C-A.

\subsection{Robotic Behavior Coordination: Skills, Tasks, World Model}

Figure 3.35 shows the recurring principle of a block with ports and a data sheet in the context of runtime behavior coordination. In its simplest form, the complete robot is represented as a block that comes with a port. That port allows to call tasks or skills out of the data sheet of the robot and thereby have them executed. Examples for its use are user interfaces to command the robot but also fleet managers, or MES (manufacturing execution systems). Tasks and skills in that data sheet of the robot are a subset of those available on the robot as some are just internally used alternatives that are presented in a more generic way outside the robot. The skills of a robot come with the software components that the system builder selected and instantiated. The system builder also selects a task set that fits to the skills. Both the skills and the tasks end up on the robot so that they can be accessed by the behavior executor. This is a (set of) software component(s) providing the runtime execution mechanism for tasks and skills. At runtime, the behavior executor comprises at least the link from the task/skill models to the software components via their behavior interfaces (the skill interface component). 


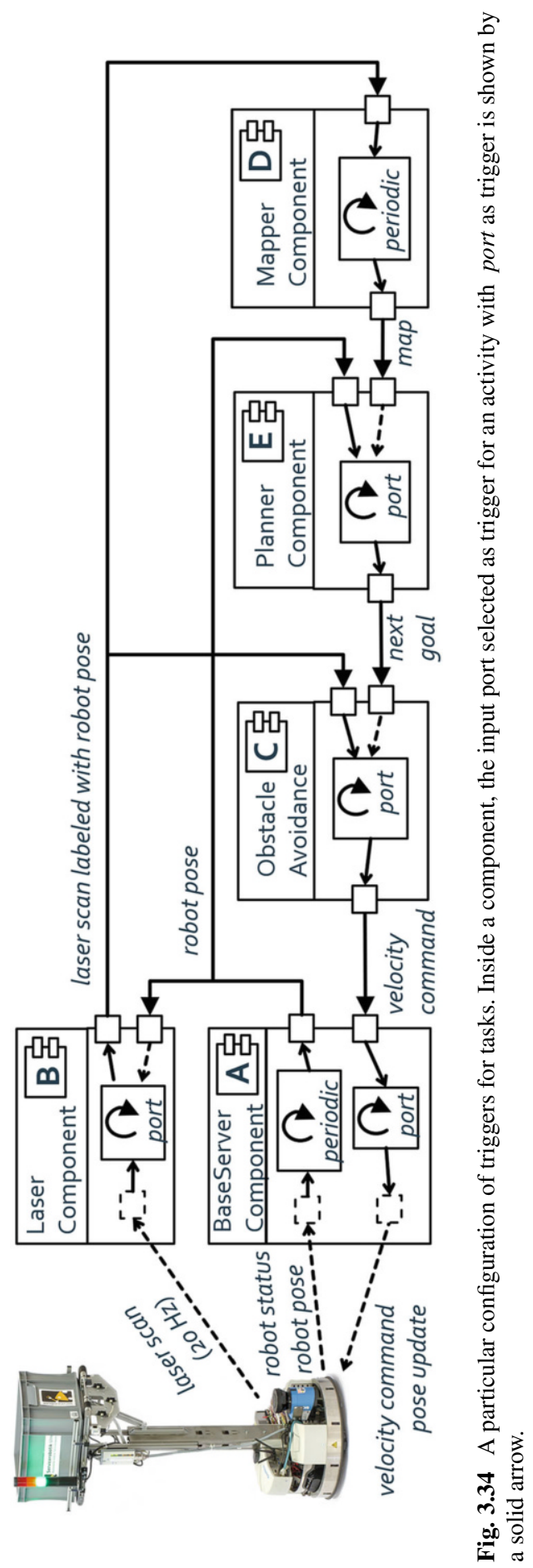




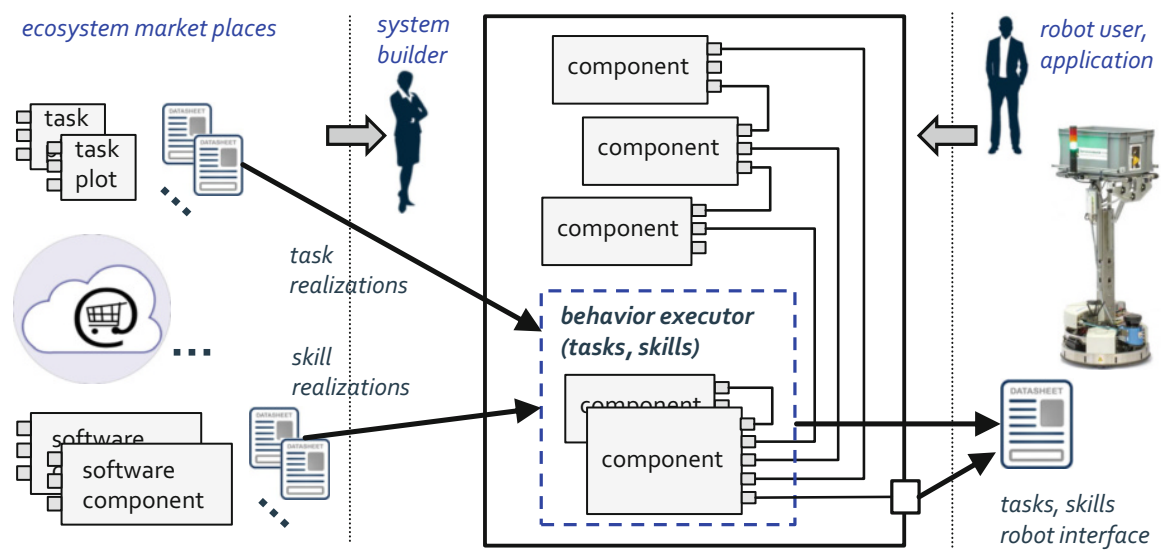

Fig. 3.35 Data sheets with tasks and skills: from the ecosystem marketplaces to their runtime execution by robots.

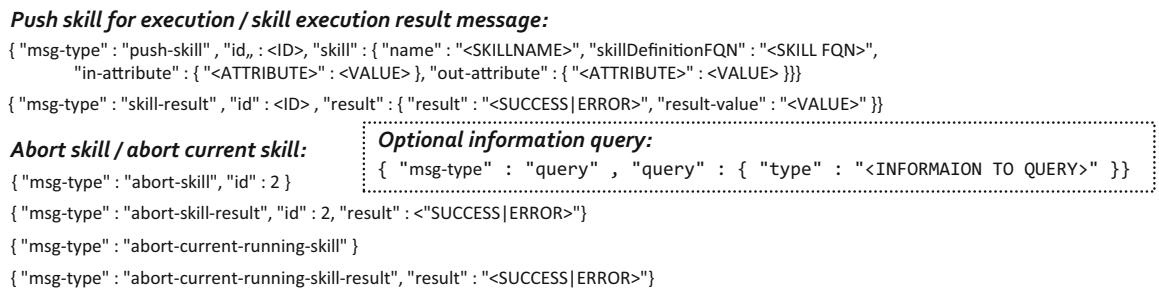

Fig. 3.36 The JSON format for commanding and querying a robot.

One implementation of the port for commanding tasks/skills to a robot uses a JSON representation (Fig. 3.36). The possible content and the parser in the skill interface component are directly derived from the data sheet of the robot.

A behavior executor can be as simple as allowing only one skill to be called at a time in order to avoid conflicts in concurrently executing skills. More advanced behavior coordination mechanisms are based on state automata or behavior trees. The tool Groot of the RobMoSys ITP MOOD2Be is one such example, which provides a dedicated graphical tool to support the behavior developer in arranging skills into behaviors. Skills can either be imported from the data sheet of a particular robotic system, or it can be the set of skills of a particular domain or even skills from different domains. The outcome is a task in the form of a behavior tree. That can be executed on any robotic system that comes with the skills referenced in the behavior tree. Typically, the behavior-tree executor is on-board the robot, becomes part of the behavior executor and uses its skill interface component. The robot becomes a block with a port where the port now forms a task-level interface.

Hierarchical task nets are another mechanism for behavior coordination. A powerful implementation is given by SmartTCL [35]. The behavior developer view within the SmartMDSD toolchain has already been introduced in Sect. 4.3. As 
shown in Fig. 3.37, hierarchical task nets allow for refinements at runtime taking into account the current situation and context. They can also deliberately include external solvers to decide between alternatives or to decide for a trade-off of different parameters. This is key when it comes to binding left-open variation points at runtime along given policies for executing tasks, even in open-ended environments, in an adequate and robust way.

Again, the robot comes with a port that allows to command tasks to the robot and the data sheet contains all the tasks that the robot accepts. All the tasks with their current execution status including their current level of refinement are present on the agenda. Tasks can also be generated from inside the robot. An example for this is a task that ensures that the robot drives to a charging station before it runs out of power. The agenda is processed by the so-called sequencer.

The core feature of the agenda-based mechanism (and of the hierarchical task nets) is the free-of-conflicts execution of concurrent activities. Hierarchical task nets go for resource reservations of their resources, and sub-tasks can only operate within the setting given by their superordinated task (horizontal/vertical interaction and composition of resource shares). For example, a task might reserve the gripper such that no other concurrent task can run concurrently that might release the carried object in the gripper. The self-model of the robot with its resources is part of the knowledge base. It gets initially filled from the data sheets of the components of the robot at the system building step.

\section{State of the Art, State of the Practice and Conclusion}

A comprehensive overview of the rich body of knowledge of different robotics software frameworks, of relevant software engineering approaches and of their applications in related domains (such as automotive, avionics, industry 4.0), of technologies (such as simulation in the loop, model-driven workbenches) and of advances in, e.g. formal methods (such as validation, verification, semantic modelling), is just impossible. Instead of just describing arbitrarily selected approaches, it is very revealing to think about how the relationship between robotics, programming, software engineering and the other just mentioned domains has changed over time.

There are all kinds of examples of robot programming. In earlier times, it was often related to bare-metal programming and could be done only by highly skilled programming experts. This approach neither scaled with respect to the complexity challenge nor allowed application domains to easily get access to and make use of robotics technology.

This was followed by a huge diversity of different robotics software frameworks, each favouring different needs and thus filling a particular niche [37, 38]. Indeed, these all can be best understood in their time since they have been heavily influenced by at least (1) the technology available and accessible at that time, (2) the kinds of robots and domains put into focus (mobile robots, manipulation, flying robots, 


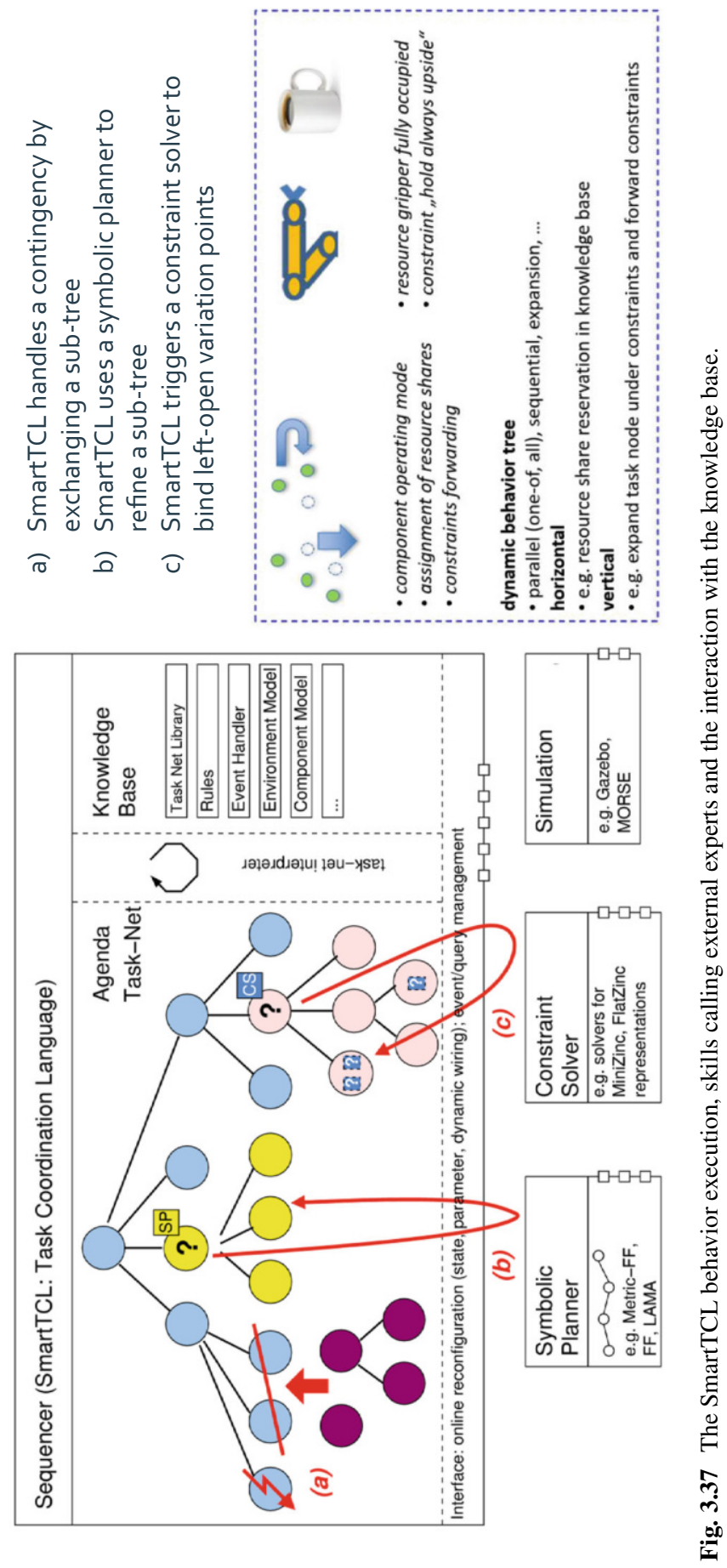


etc.), (3) whether the focus has been more on functionalities (typically driven by academia) or on (application) scenarios (typically driven by industry and also by competitions like the RoboCup) and even by (4) the (non)availability and (non)affordability of standard robot platforms (mobile robot Pioneer, humanoid robot NAO, Willow Garage PR2, PAL Robotics Tiago, FESTO Robotino, the Universal Robots UR-Arm and many others), cheap sensors (laser rangers, RGB$\mathrm{D}$ cameras, tablets for user interfaces), ubiquitous and always available Internet connections as well as enough and cheap computing power.

Robotics software has seen tremendous changes and shifts up to now. In the past, it has been left to the readers of a paper to try to implement a described algorithm on their own and it was nearly impossible to reproduce experiments. Nowadays, it is quite standard that papers are accompanied by at least prototypical and easyto-access implementations. In particular, there are more and more graphical tools to support low-code programming (e.g. for parameterization of templates for particular tasks such as palletizing, etc.).

Latest developments in software frameworks for robotics now link cloud platforms and robotic systems. Examples are the Microsoft Azure Cognitive Services and the Amazon Web Services RoboMaker. Cloud services have the potential to boost object recognition and learning algorithms as well as inference algorithms and to add these to the variety of skills of robots. Cloud services have inherent advantages when it comes to cheap processing power, sharing of data sets for learning and even sharing of experiences between robots. This then allows a robot facing a new situation to take advantage from other robots that have dealt with that situation already.

In general, there is a long-standing fruitful relationship with mutual benefits between robotics and other related domains. For example, middleware systems are researched and pushed forward by a community in their own, and robotics makes use of their outcomes. On the other hand, the middleware community is stimulated by insights and demanding needs from robotics.

Nevertheless, all interdisciplinary fields, and thus robotics as well, come with a natural and deeply rooted challenge. We make this clear by exaggerated and catchy examples. Middleware experts provide generic middleware systems to try to cover as many application needs as possible. Some robotic experts consider those middleware systems to be of unnecessary complexity and with a too big footprint. In consequence, they implement message-based blackboard communication architectures on their own and thereby step-by-step discover all the challenges already well-known (and sometimes even solved) in the competent domain.

Software engineering experts come up with more and more generic offers for modeling aspects of software systems [39]. However, they either abstract away the steps to ground the models (as this is just domain-specific) or they do it in an exemplary manner only (e.g. by low-complexity examples with educational robots). Roboticists then neither see the benefits given their complex challenges nor do they see an easy-access path.

Another example relates to the question of how to generate trust into a robotics system. Roboticists are aware of formal methods for verification, validation, 
certification, runtime sanity checks, co-simulation (even at runtime by the robot before a decision is executed) and of many others [40]. However, it is about making reasonable matches for each technology: what kind of problem and what kind of complexity can it handle, what is the effort in using it, what is the coverage and reliability and is that within the needs and the economic constraints of the application domain. Unfortunately, there are again lots of mismatches. The reason is that at least advanced robotic systems have to face additional complexity due to open worlds.

Basically, after years of huge advancements within all the different silos relevant for robotics, it is now the time to again rethink matches between requirements and now available opportunities. Neither roboticists shall become experts for those silos, nor experts from those silos shall become experts for robotic systems. Rather, achieving mutual benefits requires (1) mutual respect for the body of knowledge of different silos and domains; (2) open and honest explications of the capabilities, but also the deficiencies of the state-of-the-art in a silo; and (3) neither blaming nor getting blamed for that state of the art, but rather seeking for possible hooks and interfaces between the silos and illustrating the benefits first along low-hanging use cases instead of starting with full-blown examples and missionary work first. Although that all should be quite standard, it always is a challenge because it is about establishing and providing opportunities for fruitful interactions between different silos for the mutual benefit of all.

We consider model-driven approaches, with their now-achieved maturity level and when applied as described in this chapter, to be a particular fit to serve as a moderator for this. As outlined, model-driven approaches are not foremost just modeling everything instead of coding. They are also not just about code generation. Instead, they are the means to provide consistent links between different domains and their assets and thus can establish links between so far isolated silos. They allow to stay with presentations specific to robotics but map those in a semantically correct way onto the huge variety of offered implementational technologies.

The broadest coverage of concepts, tools, implementations and applications of model-driven software engineering in robotics is represented by the EU H2020 project RobMoSys and can be found in the RobMoSys Wiki [19, 21]. In-depth presentations of core concepts are available via $\mathrm{PhD}$ theses (some still underway) [29, 41, 42]. RobMoSys started a movement toward model-driven software engineering approaches in robotics and is continuously updated with broadest community involvement based on discussions in the related forum [43]. It also contains lots of references beyond robotics.

The euRobotics AISBL Topic Group on Software Engineering, System Integration, System Engineering is another entry point for the community. It shapes the European road-mapping in software systems engineering for robotics. It will also take over stewardship for the body of knowledge managed and organized for the community by RobMoSys, heading for the sustainability of that starting point and the related movement and also ensuring its liveliness after the runtime of the project. 
The community also gathers in the Technical Committee on Software Engineering for Robotics and Automation (IEEE RAS TC-SOFT). Further currently active community services are the MORSE (Model-Driven Robot Software Engineering) workshops, the SIMPAR (Int. Conf. on Simulation, Modeling, and Programming for Autonomous Robots) conference, the MODELS (Int. Conf. on Model Driven Engineering Languages and Systems) conference but also JOSER (Journal of Software Engineering for Robotics). Further material is also available via the Dagstuhl Seminar 17071 Computer-Assisted Engineering for Robotics and Autonomous Systems [40]. Relevant software and modeling activities with impact on robotics are also driven by the Reference Architecture Model Industrie 4.0 (RAMI), the Asset Administration Shell and related OPC UA companion specifications.

\section{References}

1. J. Stubbe, J. Mock, S. Wischmann, The Acceptance of Service Robots: Tools and Strategies for the Successful Deployment in Companies. Study commissioned by BMWi as part of the PAiCE Technology Programme (iit-Institut für Innovation und Technik in der VDI/VDE Innovation + Technik GmbH, Berlin, 2019)

2. D. Brugali (ed.) Software Engineering for Experimental Robotics. Springer Tracts in Advanced Robotics (Springer, Berlin, 2007). ISBN: 3540689494

3. M. Hägele, N. Blümlein, O. Kleine, EFFIROB - Wirtschaftlichkeitsanalysen neuartiger Servicerobotik-Anwendungen und ihre Bedeutung für die Robotik-Entwicklung (Eine Analyse der Fraunhofer-Institute IPA und ISI im Auftrag des BMBF, 2011)

4. SPARC - The Partnership for Robotics in Europe. Strategic Research Agenda (SRA) for Robotics in Europe 2014-2020. euRobotics aisbl (2013 \& 2014)

5. D. Mourtzis, Challenges and future perspectives for the life cycle of manufacturing networks in the mass customisation era. Logist. Res. 9, 2 (2016). https://doi.org/10.1007/s12159-0150129-0

6. M. Teulieres, J. Tilley, L. Bolz, P.M. Ludwig-Dehm, S. Wägner, Industrial Robotics - Insights into the Sector's Future Growth Dynamics (McKinsey \& Company, New York, 2019)

7. J.F. Moore, Predators and prey: a new ecology of competition. Harv. Bus. Rev. 71(3), 75-83 (1993)

8. E. Kelly, Business Ecosystems Come of Age. Part of the Business Trends Series (Deloitte University Press, New York, 2015). DUP_1048-Business-ecosystems-come-ofage_MASTER_FINAL.pdf

9. S. Hallsteinsen, M. Hinchey, S. Park, K. Schmid, Dynamic software product lines. Computer 41(4), 93-95 (2008)

10. J. Bosch, From software product lines to software ecosystems, in Proceedings of the 13th Int. Software Product Line Conference (2009), pp. 111-119. https://doi.org/10.1145/1753235. 1753251

11. W. Mahnke, S.-H. Leitner, M. Damm, OPC Unified Architecture (Springer, New York, 2009). ISBN: 978-3-540-68898-3

12. OPC Foundation Companion Specifications. https://opcfoundation.org/about/opctechnologies/opc-ua/ua-companion-specifications/

13. J. Bosch, P. Bosch-Sijtsema, From integration to composition: on the impact of software product lines, global development and ecosystems. J. Syst. Softw. 83(1), 67-76 (2010). ISSN:0164-1212. https://doi.org/10.1016/j.jss.2009.06.051 
14. C. Schlegel, A. Lotz, M. Lutz, D. Stampfer, J.F. Inglés-Romero, C. Vicente-Chicote, Modeldriven software systems engineering in robotics: covering the complete life-cycle of a robot. Inf. Technol. 57(2), 85-98 (2015). De Gruyter, Oldenbourg

15. L. Andrade, J.L. Fiadeiro, J. Gouveia, G. Koutsoukos, Separating computation, coordination and configuration. J. Softw. Mainten. Evol. Res. Pract. 14(5), 353-369 (2002)

16. E.A. Lee, S.A. Seshia, Introduction to Embedded Systems: A Cyber-Physical Systems Approach, 2nd edn. (MIT Press, Cambridge, 2017)

17. M. Lutz, D. Stampfer, A. Lotz, C. Schlegel, Service robot control architectures for flexible and robust real-world task execution: Best practices and patterns, in Informatik 2014, Workshop Roboter-Kontrollarchitekturen. LNI der GI (Springer, New York, 2014). ISBN:978-3-88579626-8

18. F. Buschmann, R. Meunier, H. Rohnert, P. Sommerlad, M. Stal, Pattern-Oriented Software Architecture, Volume 1, A System of Patterns (Wiley Press, Hoboken, 1996). ISBN: 978-0-47195869-7

19. RobMoSys Wiki. Cited 9. Aug 2020. https://robmosys.eu/wiki/

20. E. Scioni, N. Huebel, S. Blumenthal, A. Shakhimardanov, M. Klotzbücher, H. Garcia, H. Bruyninckx, Hierarchical hypergraphs for knowledge-centric robot systems: a composable structural meta-model and its domain specific language NPC4. JOSER - Spec. Iss. DomainSpec. Lang. Mod. Robot. Syst. 7(1), 55-74 (2016)

21. RobMoSys Wiki Modeling Section. Cited 9. Aug 2020 https://robmosys.eu/wiki/modeling:<title of subordinate document $>$

22. G. Engels, A. Schürr, Encapsulated hierarchical graphs, graph types, and meta types. Electron. Notes Theor. Comput. Sci. 2, 101-109 (1995)

23. M. Levene, A. Poulovassilis, An object-oriented data model formalised through hypergraphs. Data Knowl. Eng. 6, 205-224 (1991)

24. C.A. Szyperski, D. Gruntz, S. Murer, Component Software - Beyond Object-Oriented Programming. Addison-Wesley Component Software Series, 2nd edn. (Addison-Wesley, Boston, 2002)

25. I. Crnkovic, S. Sentilles, A. Vulgarakis, M.R.V. Chaudron, A classification framework for software component models. IEEE Trans. Softw. Eng. 37(5), 593-615 (2011). https://doi.org/ 10.1109/TSE.2010.83

26. C. Schlegel, A. Lotz, A. Steck, SmartSoft - The State Management of a Component. Technical Report 2011/01. Hochschule Ulm, Germany (2011) ISSN:1868-3452. http://www. zafh-servicerobotik.de/dokumente/ZAFH-TR-01-2011-ISSN-1868-3452.pdf

27. C. Schlegel, Navigation and Execution for Mobile Robots in Dynamic Environments: An Integrated Approach. PhD thesis, Uni Ulm (2004)

28. D. Stampfer, A. Lotz, M. Lutz, C. Schlegel, The SmartMDSD toolchain: an integrated MDSD workflow and Integrated Development Environment (IDE) for Robotics Software. JOSER Spec. Iss. Domain-Spec. Lang. Mod. Robot. Syst. 7(1), 3-19 (2016)

29. M. Lutz, Model-Driven Behavior Development for Service Robotic Systems: Bridging the Gap between Software- and Behavior-Models (work in progress)

30. A. Lotz, A. Steck, C. Schlegel, Runtime monitoring of robotics software components: increasing robustness of service robotic systems, in International Conference on Advanced Robotics (ICAR '11), Tallinn, Estonia (2011)

31. GitHub Repository with API specifications. Cited 9. Aug 2020. https:/github.com/ Servicerobotics-Ulm/SmartSoftComponentDeveloperAPIcpp

32. C. Schlegel, A. Lotz, ACE/SmartSoft - Technical Details and Internals. Technical Report 2010/01, Hochschule Ulm, Germany (2010). ISSN:1868-3452. http://www.zafhservicerobotik.de/dokumente/ZAFH-TR-01-2010-ISSN-1868-3452.pdf

33. A. Lotz, A. Hamann, R. Lange, C. Heinzemann, J. Staschulat, V. Kesel, D. Stampfer, M. Lutz, C. Schlegel, Combining robotics component-based model-driven development with a model-based performance analysis, in Proceedings of the IEEE International Conference on Simulation, Modeling, and Programming for Autonomous Robots (SIMPAR) (2016), pp. 170176 
34. The Eclipse-based Open-Source SmartMDSD Toolchain. Cited 9. Aug 2020. https://wiki. servicerobotik-ulm.de/getting-started-guide. https://projects.eclipse.org/projects/modeling. smartmdsd

35. A. Steck, C. Schlegel, Managing execution variants in task coordination by exploiting designtime models at runtime, in Proceedings of the IEEE/RSJ International Conference on Robotics and Intelligent Systems (IROS), San Francisco, USA, September (2011)

36. SmartMDSD Tutorials. Cited 9. Aug 2020 https://wiki.servicerobotik-ulm.de/tutorials:<title of subordinate document>

37. A. Elkady, T. Sobh, Robotics middleware: A comprehensive literature survey and attributebased bibliography. J. Robot. (2012). Article ID 959013. https://doi.org/10.1155/2012/959013. https://core.ac.uk/download/pdf/52956509.pdf

38. A. Ramaswamy, B. Monsuez, A. Tapus, Model-driven software development approaches in robotics research, in Proceedings of the 6th International Workshop on Modeling in Software Engineering (MiSE), June (2014), pp. 43-48. https://doi.org/10.1145/2593770.2593781

39. D. Akdur, V. Garousi, O. Demirors, A survey on modeling and model-driven engineering practices in the embedded software industry. J. Syst. Arch. (2018). https://doi.org/10.1016/j. sysarc. 2018.09.007

40. E. Abraham, H. Kress-Gazit, L. Natale, A. Tacchella (organizers), Computer-Assisted Engineering for Robotics and Autonomous Systems. Dagstuhl-Seminar 17071, 12. - 17.02.2017. https://www.dagstuhl.de/17071

41. D. Stampfer, Contributions to System Composition using a System Design Process driven by Service Definitions for Service Robotics. PhD thesis, Technische Uni München (2018)

42. A. Lotz, Managing Non-Functional Communication Aspects in the Entire Life-Cycle of a Component-Based Robotic Software System. PhD thesis, Technische Uni München (2018)

43. The RobMoSys Discourse Forum. Cited 9. Aug 2020. https://discourse.robmosys.eu/

Open Access This chapter is licensed under the terms of the Creative Commons Attribution 4.0 International License (http://creativecommons.org/licenses/by/4.0/), which permits use, sharing, adaptation, distribution and reproduction in any medium or format, as long as you give appropriate credit to the original author(s) and the source, provide a link to the Creative Commons license and indicate if changes were made.

The images or other third party material in this chapter are included in the chapter's Creative Commons license, unless indicated otherwise in a credit line to the material. If material is not included in the chapter's Creative Commons license and your intended use is not permitted by statutory regulation or exceeds the permitted use, you will need to obtain permission directly from the copyright holder.

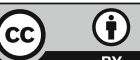

